\title{
Funktionsprïfungen der Leber in Graviditate, sub partu, im Wochenbett und bei Eklampsie. - Ein Beitrag zur Frage der Sehwangerschaftsleber.
}

\author{
Von \\ cand. med. B. Walthard. \\ (Aus der Universitäts.Frauenklinik zu Frankfurt a. M. [Direktor: Geheimrat \\ Prof. Dr. Seitz].) \\ (Eingegangen am 2\%. Oktober 1921.)
}

In Deutschland war Hofbauer der erste, der sich der Ansicht der Franzosen betreffs der Insuffisance hépatique in der Schwangerschaft restlos anschloß. Er fußte vornehmlich auf pathologisch-anatomischen Argumenten, betonte indessen auch schon die Funktionsstörung im Sinne einer Kohlenhydrat- (Lävuloseprobe), Eiweil- and Fettstoffwechselstörung. Die Schwangerschaftsleber in diesem Sinne wurde von Schikkele bestritten; Heinrichsdorf nahm einen vermittelnden Standpunkt ein. Die pathologisch-anatomische Frage der Schwangerschaftsleber ist heute noch nicht restlos gelöst.

Funktionsprüfungen der Leber sind viele ausgeführt worden. Die früheren Untersuchungen leiden alle darunter, daß man den Harn als Indikator von Leberfunktionsstörungen untersuchte, ohne indessen über die jeweilige Rolle der Niere einen Aufschluß zu haben. Blutuntersuchungen in größerem Maßstabe konnten wegen der großen Mengen Blut, die man für eine Untersuchung benötigte, nicht ausge. führt werden.

Erst die Einfuhrung von Mikromethoden, insbesondere der Bangschen, gestattete, in größerem Umfange Blutuntersuchungen vorzunehmen und aus ihnen auf die Funktion der Leber Rückschlüsse zu ziehen.

Mit diesen Methoden wurde in den Jahren 1918-1920 am physiologisch-chemischen Laboratorium der Universitätsfrauenklinik zu Frankfurt a. M. in mehreren Untersuchungsreihen die Funktion der Leber während.der Schwangerschaft, sub partu und im Wochenbett, sowie bei Eklampsie geprüft. Toh werde jeweils, eingangs der einzelnen Funktionsprüfungen, die schon auf diesem Gebiete ausgeführten Arbeiten zusammenfassend erwähnen. 
Unsere Untersuchungen wurden angestellt teils zur Nachprüfung schon bestehender Arbeiten, teils zu Versuchen, neues Licht in die Vorgänge des Leberstoffwechsels während der Gravidität und im Wochenbett werfen zu können.

Wir bedienten uns dabei folgender Methoden:

1. Bestimmung des Blutzuckerspiegels;

2. Bestimmung des Speicherungsvermögens der Leber für Traubenzucker bei intravenösen Infusionen von $500 \mathrm{ccm} 5,4$ proz. Dextrose oder Lävuloselösung;

3. Bestimmung des Mobilisierungsvermögens der Leber für Traubenzucker durch Injektionen von 0,3 oder meist $0,75 \mathrm{ccm}$ Adrenalin;

4. a) Urobilingehalt des Urins,

b) Einfluß einer Injektion von $0,75 \mathrm{ccm}$ Adrenalin auf den Urobilinspiegel des Urins;

5. Bestimmung des Reststickstoffes des Blutes.

Sämtliche mikrochemischen Untersuchungen wurden von Frl. Pa ula Joos, Laboratoriumsassistentin an der Universitätsfrauenklinik, ausgeführt.

Im folgenden werden die einzelnen Methoden besprochen werden.

Der Blutzuckerspiegel in der Gravidität, im Wochenbett und bei Eklampsie.

Der Blutzuckerspiegel schwankt beim gesunden Menschen zwischen $0,07 \%$ und $0,11 \%$, Bang. In der Regel hält er sich um $0,09 \%$. Werte darüber nähern sich der oberen Grenze, Werte darunter gehören zu den tiefen Normalwerten.

Das Verhalten des Blutzuckerspiegels in der Gravidität, sub partu, im Wochenbett und bei Eklampsie ist schon verschiedentlich Gegenstand der Untersuchung gewesen.

So fand Benthin während der Schwangerschaft einen eher tiefen Stand des Blutzuckerspiegels, ganz besonders in der zweiten Hälfte der Gravidität. Sub partu kommt es zu einer Geburtshyperglykämie, die im Wochenbett rasch wieder zur Norm absinkt. Benthi erklärt diese Ausschwemmung von Zucker ins Blut als Reaktion der Leber auf vermehrte Muskelarbeit, Muskelhunger. Auch bei Eklampsie fand er eine Hyperglykämie, die er durch die vermehrte Muskelarbeit und Krämpfe zustande gekommen denkt. Neubauer und Novac konnten die Resultate Benthins im allgemeinen bestätigen. Auch Ryser kam zu gleichen Ansichten wie die anderen Autoren.

\section{Eigene Untersuchungen.}

Bei unseren Untersuchungen bedienten wir uns der Bangschen Mikromethode zur Bestimmung des Blutzuckergehaltes. Die Werte sind sämtliche Nüchternwerte. Das Blut wurde des Morgens vor dem 
Frühstück aus dem Finger entnommen. Wir teilten das uns zu Gebote stehende Material in drei Gruppen ein:

1. Normale Gravidität, Partus und Wochenbett;

2. Gravidität und Wochenbett bei verschiedenen Erkrankungen;

3. Eklampsien.

1. a) Normale Gravidität: Unsere Werte schwanken um $0,07 \%$. Es ist deutlich ersichtlich, daß der Blutzuckerspiegel gegen den Partus zu steigt bis $0,09 \%$ (Stumpf), am letzten Tage vor dem Partus. Im Gegensatz dazu ist $0,056 \%$ (Schneble) der tiefste Wert zwei Monate vor dem Partus, was dem tiefen Stand des Blutzuckerspiegels in den letzten Monaten ante partum entspricht. Der eine Wert von 0,115 bei (Schneble) ist nicht zu werten, da er keinen Nüchternwert darstellt. Unsere Fälle beweisen also von neuem die Richtigkeit der von Benthin zuerst aufgestellten Tatsache, daß der Blutzuckerspiegel in den letzten Monaten ante partum tiefe Werte aufweist. In den letzten Tagen ante partum erhebt sich der Spiegel wieder zur Norm.

b) Sub partu: Kommt es zu einer Hyperglykämie oder zu Werten an der oberen Grenze des Normalen, wie 2 Fälle (Müller, Dörr) deutlich beweisen (Bestimmungen am Tage der Geburt).

Wie kommt es, daß der Blutzuckerspiegel sich von den tiefen Werten in den letzten Schwangerschaftsmonaten über die Norm von $0,09 \%$ hinaus bis zu Werten von über $0,1 \%$ erhebt? Nach der Ansicht von Benthin, der eine vermehrte Muskelarbeit als Grund der Zuckerausschüttung ansieht, wäre dieser, stete Anstieg in einer in den letzten Wochen ante partum einsetzenden Verstärkung der Schwangerschaftswehen zu finden, die dann in die Geburtswehen übergehen und die eigentliche Hyperglykämie zur Folge haben. Auch müßte nach seiner Ansicht bei langdauernden Geburten, bei Geburten mit Hindernissen der Blutzuckerspiegel beträchtlich erhöht sein. Auch bei Eklampsie, wo es zu Muskelkrämpfen kommt, müßte eine besonders starke Ausschüttung von Zucker ins Blut erfolgen. Indessen betonte Benthin in seiner Arbeit, die Höhe des Blutzuckerspiegels steht in keinem $\mathrm{Zu}$ sammenhang mit der Dauer der Geburt, $d . h$. besonders vermehrte Muskelarbeit führt nicht zu besonders vermehrter Ausschüttung. Ferner erklärte er das Steigen des Spiegels trotz Mehrverbrauch von Zucker durch den Muskel als reichlichere Ausschüttung von Zucker ins Blut, als der Muskel momentan verarbeiten kann.

Wie verhält sich nun bei Nichtgraviden der Blutzuckerspiegel bei vermehrter Muskelarbeit? Es liegt eine Arbeit von Grote und eine von Brosamlen und Sterkel vor. Beide konnten zeigen, daß Muskelarbeit den Zuckergehalt des Blutes nicht nur nicht erhöht, sondern eher erniedrigt. Momentane Steigerung des Spiegels unmittelbar nach Beginn der Arbeit kann vorkommen, er sinkt aber sehr rasch wieder 
zur Norm und sogar unter die Norm. Der Blutzuckerspiegel sub partu ist während des ganzen Partus, ja noch nach dem Partus erhöht, das ich aus neueren, noch nicht veröffentlichten Untersuchungen an der Zürücher Frauenklinik durchaus bestätigt fand.

Dadurch verliert die Ansicht Benthins und Rysers, die Hyperglykämie sub partu sei durch die Muskelarbeit bedingt, an Wahrscheinlichkeit.

Gro te schreibt die Hyperglykämie sub partu zum Teil der Dyspnöe sub partu zu, die als Folge einer Kohlensäureüberladung des Blutes eintritt.

Ferner darf die Möglichkeit einer gewissen Schwäche der Leber sub partu zugegeben werden. Grote und Brosa mlen zeigten, daß beim Diabetiker der Blutzuckerspiegel bei Arbeit stieg. Grote verglich nun diese Diabetikerkurve mit den Werten, die er bei Erkrankungen der Leber erhielt. Er fand, daß die Zuckerwerte bei Arbeit ebenfalls ansteigend waren und sich ähnlich wie die Diabetikerwerte verhielten. Es handelte sich also in beiden Fällen um eine erhöhte Reizbarkeit der Leber für Glykogenolyse, Lichtwitz, oder im Sinne Noordens um ein Mißverhältnis zwischen Muskelreiz bei Arbeit und Reaktion der Leber mit Ausschüttung von Zucker ins Blut. In diesem Sinne könnte man auch sub partu von einem Einfluß der Muskelarbeit sprechen. Indessen ist alsdann nicht abnormer Muskelzuckerhunger, sondern eine abnorme Reaktion der Leber auf den normalen Reiz, Noorden, das ausschlaggebende Moment.

Auch diese Auffassung steht keineswegs fest, hat aber eine gewisse Berechtigung an der Hand der Resultate von Grote und verschiedener anderer Leberfunktionsschädigungen sub partu, von denen wir im nachfolgenden sprechen werden.

c) Im Wochenbett fällt der Blutzuckerspiegel rasch wieder zur Norm ab. Unser Mittelwert beträgt 0,08\%, was wieder völlig ins Gebiet des Normalen fällt. Kurz, post partum findet man einen etwas erhöhten Spiegel, in seltenen Fällen verzögert sich das Absinken über mehrere Tage hin. Der Harnzucker blieb in der Gravidität, wie auch im Wochenbett in den meisten Fällen negativ.

2. Gravidae und Wöchnerinnen mit verschiedenen Erkrankungen: In einer Zahl von 10 Fällen hatten wir Gelegenheit zu untersuchen, wie sich der Einfluß verschiedener Erkrankungen in graviditate und im Wochenbett auf den Blutzuckerspiegel geltend machte und zwar 2 mal bei Hydrops gravidarum im 9 . Schwangerschaftsmonat; 3 mal bei Nephropathia gravidarum ohne Eklampsie, 1 mal bei chronischer Nephritis, $3 \mathrm{mal}$ bei Icterus in graviditate und $2 \mathrm{mal}$ bei Chorea gravidarum; schließlich bei einer Osteopathie kombiniert mit Gravidität (siehe S. 73, Tab. Ib).

Aus der Tabelle ist ersichtlich, daß in der Mehrzahl der Fälle unter 
Berücksichtigung der oben erwähnten Geburtshyperglykämie (Dörr) die angeführten Krankheiten ohne Einfluß auf die Höhe des Blutzuckerspiegels blieben.

Einzig in einem Falle von Chorea gravidarum finden wir abnorm hohen Spiegel. Ein anderer Fall von Chorea zeigt dagegen kurz post operationem schon wieder einen normalen Spiegel.

Auch die Wochenbettswerte dieser Kranken sind durchaus normal.

3. Eklampsien: Bei Eklampsie konamt es kurz ante, sub und post partum zu einer Hyperglykämie. Sub partu werden die höchsten Werte erreicht; welche im Wochenbett bald schneller, bald langsamer zur Norm zurückkehren. Es ist wohl anzunehmen, daß der Grund der Zuckerausschüttung nicht in der vermehrten Muskelarbeit zu suchen ist. Wäre die vermehrte Muskelarbeit der Grund des rohen Blutzuckerspiegels, so müßte man um so höheren Blutzuckerspiegel erhalten, je mehr Anfälle die Patientin erlitten hätte. Vergleichen wir die am Tage des Partus gefundenen Zuckerwerte mit der Anzahl der Anfälle an diesem Tage, so finden wir für:

\begin{tabular}{|c|c|c|c|c|c|}
\hline & nfälle & nen & Wert & von. & 0,099 \\
\hline & nfall & $"$ & , & , & $0,139 \%$ \\
\hline & Anfälle & "y & ", & , & $0,114 \%$ \\
\hline 16 & " & $"$ & $"$ & & $0,127 \%$ \\
\hline & " & " & $"$ & t & $0,127 \%$ \\
\hline & & $"$ & $"$ & $"$ & $0,165 \%$ \\
\hline
\end{tabular}

Aus unserer Tabelle geht hervor, daß es bei vielen Anfällen zu niedrigen unteren und erhöhten Werten, bei wenig Anfällen zu sehr hohen und mäßig erhöhten Werten kommen kann. Dieser Wechsel läßt sich eher als Gradmesser der Stärke der Lebertoxikosen verwerten, ganz unabhängig von der Zahl der Anfälle. Ebenso ist wohl das Tempo des Absinkens des erhöhten Blutzuckerspiegels zur Norm im Wochenbett ein Maßstab für die Schwere der Leberschädigung, wie dies bei Erkrankungen der Leber aus extragenitaler Ursache nachgewiesen wurde, Ta $n n$ h a user und Pfitzer, Tacha u. Die vielen Wochenbettswerte aufTab.Ic, S. 74-76 beweisen klar, daß der Spiegel schließlich wieder zur Norm zurückkehrt (vgl. die Fälle Streitenberger oder Lautz).

Der Harnzucker ist nur in wenigen Fällen positiv. Gerade bei hohem Spiegel sieht man oft ein dichtes Nierenfilter für Zucker (vgl. Tab. Ic, S. $74-76)$.

\begin{tabular}{lccc}
\multicolumn{4}{c}{ Tabelle Ia. Blutzackerspiegel. } \\
Normale Gravidität und Wochen bett. \\
Name \\
Trub & Datum & $\begin{array}{c}\text { Blutzuckergehal in \%, } \\
\text { nüchterne Werte }\end{array}$ & Urin-Sach. \\
& 8. XII. 1919 & 0,071 & neg. \\
10. XII. 1919 & partus & \\
11. XII. 1919 & 0,086 & neg. \\
17. XII. 1919 & 0,079 & neg.
\end{tabular}


Tabelle I a (Fortsetzung).

\begin{tabular}{|c|c|c|c|}
\hline Name & Datum & $\begin{array}{l}\text { Blutzuckergehalt in } \% \text {, } \\
\text { nüchterne Werte }\end{array}$ & Urin-Sach \\
\hline \multirow[t]{3}{*}{ Grund } & 19. II. 1920 & 0,073 & neg. \\
\hline & 24. II. 1920 & partus & \\
\hline & 27. II. 1920 & 0,064 & nfg. \\
\hline \multirow[t]{3}{*}{ Bücher } & 18. II. 1920 & 0,088 & neg, \\
\hline & 19. II. 1920 & partus & \\
\hline & 20. II. 1920 & 0,099 & neg. \\
\hline \multirow[t]{2}{*}{ Aberle } & 23. II. 1920 & 0,071 & neg. \\
\hline & $\begin{array}{l}\text { 20. II. } 1920 \\
\text { 2. III. } 1920\end{array}$ & $\begin{array}{l}\text { partus } \\
0,078\end{array}$ & neg. \\
\hline \multirow[t]{3}{*}{ Lippert } & 26. XI. 1919 & 0,059 & neg. \\
\hline & 27. XI. 1919 & 0,073 & neg. \\
\hline & 15. XII. 1919 & partus & \\
\hline \multirow[t]{5}{*}{ Schneble } & 8. IX. 1919 & 0,056 & neg. \\
\hline & 18. IX. 1919 & 0,115 kein Nüchtern $W$. & pos. \\
\hline & 7. XI. 1919 & partus & \\
\hline & 15. XI. 1919 & 0,066 & neg. \\
\hline & 20. XI. 1919 & 0,073 & neg. \\
\hline \multirow[t]{3}{*}{ Stumpf } & 20. X. 1919 & 0,090 & pos. \\
\hline & 21. X. 1919 & partus & \\
\hline & ¿1. X. 1919 & 0,071 & pos. \\
\hline \multirow[t]{2}{*}{ Müller } & 19. X. 1919 & partus & \\
\hline & 19. X. 1919 & 0,106 & neg. \\
\hline \multirow[t]{2}{*}{ Döhler } & 17. X. 1919 & partus & \\
\hline & 18. X. 1919 & 0,075 & neg. \\
\hline \multirow[t]{3}{*}{ Mackloff } & 24. X. 1919 & F. partus $30 . \mathrm{W}$. & \\
\hline & 31. X. 1919 & 0,097 & neg. \\
\hline & 5. XI. 191! & 0,096 & neg. \\
\hline \multirow[t]{3}{*}{ Willenbücher } & 13. X. 1919 & partus & . \\
\hline & 14. X. 1919 & 0,091 & neg. \\
\hline & 15. X. 1919 & 0,077 & neg. \\
\hline
\end{tabular}

Tabelle Ih.

Gravidae und Wöchnerinnen mit verschiedenen Erkrankungen.

Name

Datum

Geyer

16. X. 1920

17. X. 1920

24. X. 1920

Palitza

15. VII. 1919

Hörlein

14. IV. 1920

3. V. 1920

Dörr
27. XI. 1919

27. XI. 1919

28. XI. 1919
Traubenzuckergehalt in \%, nüchterne Werte

Urin-Sach.

0,068 Hydrops 9. mens neg. 0,073 partus

0,060 Hydrops 9. mens neg. F. partus 38. W.

0,088 Chron. neph. neg.

partus

0,110 neph.

0,098 pos. Läv. pos. 


\begin{tabular}{|c|c|c|c|}
\hline Name & $\begin{array}{l}\text { Tabelle } \\
\text { Datum }\end{array}$ & $\begin{array}{l}\text { b (Fortsetzung). } \\
\text { Traubenzuckergehalt in \%: } \\
\text { nüchterne Werte }\end{array}$ & Urin-Sach. \\
\hline Schläfer & $\begin{array}{l}\text { 3. V. } 1920 \\
\text { 10. V. } 1920 \\
\text { 11. V. } 1920\end{array}$ & $\begin{array}{l}\text { F. partus } 30 . W . \\
0,089 \text { neph. } \\
0,089\end{array}$ & $\begin{array}{l}\text { neg. } \\
\text { neg. }\end{array}$ \\
\hline Weiss & 25. VI. 1919 & 0,093 A. P. neph. & neg. \\
\hline Bienmüller & $\begin{array}{l}\text { 8. I. } 1920 \\
\text { 9. I. } 1920 \\
\text { 3. II. } 1920\end{array}$ & $\begin{array}{l}0,067 \text { Gallenl. } \\
0,065 \\
\text { partus }\end{array}$ & $\begin{array}{l}\text { neg. } \\
\text { neg. }\end{array}$ \\
\hline Bachert & $\begin{aligned} \text { 13. XI. } 1919 \\
\text { 6. I. } 1920 \\
\text { 22. I. } 1920\end{aligned}$ & $\begin{array}{l}0,077 \text { icterus } \\
\text { partus } \\
0,068\end{array}$ & $\begin{array}{l}\text { neg. } \\
\text { pos. }\end{array}$ \\
\hline Guggelsberger & $\begin{aligned} & \text { 20. } \text { X. } 1919 \\
& \text { 1. XI. } 1919 \\
& \text { 3. XI, } 1919\end{aligned}$ & $\begin{array}{l}\text { abortus mens } 4 \\
0,074 \text { icterus } \\
0,083\end{array}$ & $\begin{array}{l}\text { neg. } \\
\text { neg. }\end{array}$ \\
\hline Gretzler & $\begin{array}{l}\text { 10. XI. } 1919 \\
\text { 12. XI. } 1919\end{array}$ & $\begin{array}{l}0,087 \text { icterus abort. } \\
0,092\end{array}$ & $\begin{array}{l}\text { neg. } \\
\text { neg. }\end{array}$ \\
\hline Heuss & 20. II. 1920 & 0,125 Chorea mens 8 & neg. \\
\hline Lehmkuhl & $\begin{array}{l}\text { 4. III. } 1920 \\
\text { 4. III. } 1920 \\
\text { 5. III. } 1920\end{array}$ & $\begin{array}{l}\text { sectio } \\
0,085 \text { Chorea } \\
0,083\end{array}$ & $\begin{array}{l}\text { neg. } \\
\text { neg. }\end{array}$ \\
\hline Hartmann & $\begin{array}{l}\text { 21. VII. } 1919 \\
\text { 22. VII. } 1919 \\
\text { 23. VII. } 1919\end{array}$ & $\begin{array}{l}0,067 \text { osteopath. mens } 7 \\
0,067 \\
\text { partus }\end{array}$ & $\begin{array}{l}7 \text { neg. } \\
\text { neg. }\end{array}$ \\
\hline
\end{tabular}

Tabelle Ic.

Eklampsie.

Name

Gretschel

Datum

9. IX. 1919

10. IX. 1919

12. IX. 1919

7. X. 1919

9. X. 1919

Zimmer

4. XI. 1919

5. XI. 1919

6. XI. 1919

24. XI. 1919

Kirchner

9. XII. 1919

9. XII. 1919

10. XII. 1919

Streitenberger 25. VIII. 1919

26. VIIT. 1919

28. VIII. 1919

9. IX. 1919

15. IX. 1919
Traubenzuckergehalt in \%, nüchterne Werte partus 0,099 0,097 0,085 0,094

Urin-Sach.

partus

0,073

0,095

0,070

partus

0,099

0,094

neg.

neg.

partus

0,100 neg.

0,083 neg.

0,065 neg.

0,072 neg. 
Tabelle Ic (Fortsetzung).

\begin{tabular}{|c|c|c|c|}
\hline Name & $\begin{array}{r}\text { Traub } \\
\text { I }\end{array}$ & enzuckergehalt in $\%$, & Urin-Sach. \\
\hline Riehl & $\begin{array}{l}\text { 1. IX. } 1919 \\
\text { l. IX. } 1919\end{array}$ & $\begin{array}{l}\text { partus } \\
0,139\end{array}$ & pos. \\
\hline Kümpel & $\begin{array}{l}\text { 12. XII. } 1919 \\
\text { 12. XII. } 1919 \\
\text { 13. XII. } 1919\end{array}$ & $\begin{array}{l}\text { partus } \\
0,114 \\
0,116\end{array}$ & $\begin{array}{l}\text { neg. } \\
\text { neg. }\end{array}$ \\
\hline Itiven & $\begin{array}{l}\text { 23. V. } 1919 \\
\text { 24. V. } 1919 \\
\text { 26. } \\
\text { V. } 1919\end{array}$ & $\begin{array}{l}\text { partus } \\
0,117 \\
0,108\end{array}$ & $\begin{array}{l}\text { neg. } \\
\text { neg. }\end{array}$ \\
\hline Jung & $\begin{array}{l}\text { 26. bis } 27 . \text { VI. } 1919 \\
\text { 28. VI. } 1919 \\
\text { 17. VII. } 1919\end{array}$ & $\begin{array}{l}\text { partus } \\
0,075 \\
0,084\end{array}$ & $\begin{array}{l}\text { neg. } \\
\text { neg. }\end{array}$ \\
\hline Burkhard & 30. V. 1919 & 0,127 kurz A. P. & neg. \\
\hline Lipp & $\begin{array}{l}\text { 27. VI. } 1919 \\
\text { 28. VI. } 1919\end{array}$ & $\begin{array}{l}0,127 \\
\text { partus }\end{array}$ & neg. \\
\hline Lautz & $\begin{array}{l}\text { 20. VIII. } 1919 \\
\text { 21. VIII. } 1919 \\
\text { 25. VIII. } 1919 \\
\text { 1. IX. } 1919 \\
\text { 2. IX. } 1919\end{array}$ & $\begin{array}{l}\text { partus } \\
0,165 \\
0,106 \\
0,102 \\
0,070\end{array}$ & $\begin{array}{l}\text { neg. } \\
\text { pos. } \\
\text { pos. } \\
\text { neg. }\end{array}$ \\
\hline Rein & $\begin{array}{l}\text { 11. IX. } 1919 \\
\text { 12. IX. } 1919 \\
\text { 13. IX. } 1919\end{array}$ & $\begin{array}{l}\text { partus } \\
0,068 \\
0,055\end{array}$ & $\begin{array}{l}\text { neg. } \\
\text { neg. }\end{array}$ \\
\hline Baschnagel & $\begin{aligned} & \text { 20. } \text { IX. } 1919 \\
& 24 . \text { IX. } 1919 \\
& \text { 25. } \text { IX. } 1919 \\
& \text { 8. X. } 1919 \\
& \text { 9. X. } 1919\end{aligned}$ & $\begin{array}{l}\text { partus } \\
0,082 \\
0,088 \\
0,076 \\
0,083\end{array}$ & $\begin{array}{l}\text { neg. } \\
\text { neg. } \\
\text { neg. } \\
\text { neg. }\end{array}$ \\
\hline Funk & $\begin{array}{l}\text { 28. XI. } 1919 \\
\text { 29. XI. } 1919 \\
\text { 1. XII. } 1919\end{array}$ & $\begin{array}{l}\text { sectio } \\
0,076 \\
0,084\end{array}$ & $\begin{array}{l}\text { neg. } \\
\text { neg. }\end{array}$ \\
\hline Horschler & $\begin{array}{l}\text { 12. II. } 1920 \\
\text { 24. II. } 1920\end{array}$ & $\begin{array}{l}\text { partus } \\
0,085\end{array}$ & neg. \\
\hline Schäfer & $\begin{array}{l}\text { 19. III. } 1920 \\
\text { 26. III. } 1920 \\
\text { 27. III. } 1920\end{array}$ & $\begin{array}{l}\text { sectio } \\
0,062 \\
0,086\end{array}$ & $\begin{array}{l}\text { neg. } \\
\text { neg. }\end{array}$ \\
\hline Heeb & $\begin{array}{l}\text { 22. IV. } 1920 \\
\text { 23. IV. } 1920 \\
\text { 26. IV. } 1920\end{array}$ & $\begin{array}{l}\text { partus } \\
0,064 \\
0,068\end{array}$ & $\begin{array}{l}\text { neg. } \\
\text { pos. Läv }\end{array}$ \\
\hline Swobada & $\begin{array}{l}\text { 11. IV. } 1920 \\
\text { 13. IV. } 1920 \\
\text { 14. IV. } 1920\end{array}$ & $\begin{array}{l}\text { partus } \\
0,066 \text { Ekl. post part. } \\
0,067\end{array}$ & $\begin{array}{l}\text { pos. } \\
\text { neg. }\end{array}$ \\
\hline Käkel & $\begin{array}{l}\text { 24. II. } 1920 \\
\text { 12. III. } 1920 \\
\text { 13. III. } 1920\end{array}$ & $\begin{array}{l}\text { sectio F. partus } 38 . \mathrm{W} . \\
0,081 \\
0,072\end{array}$ & $\begin{array}{l}\text { neg. } \\
\text { neg. }\end{array}$ \\
\hline
\end{tabular}




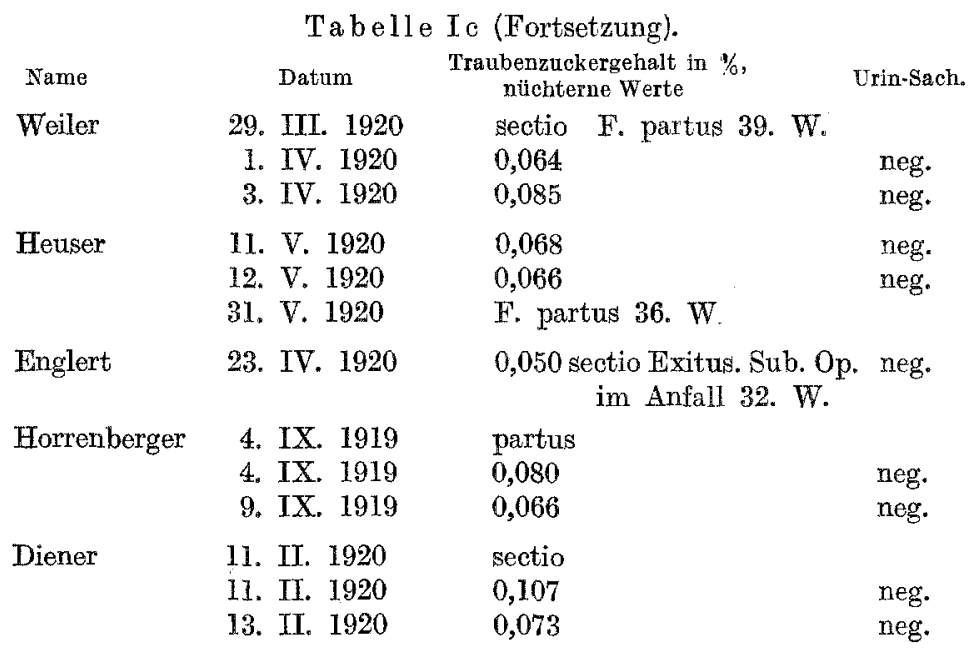

\section{Intravenöse Zuckerinfusionen.}

Die folgenden Untersuchungen über die Leberfunktion befassen sich damit, festzustellen, wie sich die Leber in ihrem Speicherungsvermögen zu Dextrose und Lävulose verhält. Ähnliche Untersuchungen sind schon gemacht worden und zwar durch Verabreichung von Dextrose oder Lävulose per os. Man fand in einigen Fällen Glykosurie, in anderen nicht, weiter Hyperglykämie, die bald wieder (spätestens in 4 Stunden) zur Norm zurückkehrte, Jakobsen, Kahler, Ryser.

Auch intravenöse Infusionen von Dextrose und Lävulose bei Nichtschwangeren und Schwangeren wurden ausgeführt und zeigten, daß nach einer Stunde, meist schon nach 15 Minuten die Speicherung des Zuckers in der Leber erfolgt war und der Spiegel wieder einen normalen Wert inne hatte, Ryser.

\section{Eigene Untersuchungen.}

Wir bedienten uns einer 5,4 proz. Traubenzucker. oder Lävuloselösung, die innerhalb 15 Minuten in die Armvene infundiert wurde. Untersuchungen an lebergesunden Nichtschwangeren haben uns ergeben, daB in spätestens einer Stunde die Leber die infundierte Zuckerlösung dem Blute entzogen und gespeichert haben soll. Zuerst wurde der Nüchternwert bestimmt, dann infundiert und nach einer Stunde von neuem der Blutzuckerwert bestimmt. Als Methode bedienten wir uns auch hier der Mikromethode von Bang.

Das Material wurde in drei Gruppen eingeteilt:

1. Gravidae und Wöchnerinnen;

2. Gravidae und Wöchnerinnen mit verschiedenen Erkrankungen;

3. Eklampsien. 
1. Gravidae und Wöchnerinnen: Die wenigen Fälle, die untersucht wurden, zeigen ein gutes Vermögen, den infundierten Traubenzucker zu speichern. Oft geht die Tendenz der Leber zu speichern so weit, daß wir einen etwas tieferen Spiegel erhalten, als dem Ausgangswert entsprach. (Schneble) und (Trub) zeigen eine leichte Verzögerung in der Speicherung einen Tag post partum, was auf eine leichte Schwäche der Leber schließen läßt.

Indessen sind die Verzögerungen gering und die Spiegelhöhe bält sich durchaus nicht in pathologischen Höhen.

Untersuchungen sub partu stehen leider noch aus und wären wichtig, die Frage des Blutzuckerspeicherungsvermögens sub partu erhellen zu kötnnen.

2. Gravidae und Wöchnerinnen mit verschiedenen Erkrankungen: Folgendè Gravidae und Wöehnerinnen mit verschiedenen Erkrankungen wurden hinsichtlich des Speicherungsvermögens der Leber für Traubenzucker geprüft:

I. 2 Fälle von Hydrops zeigten durchaus normales Sinken des Spiegels nach Infusion.

II. 2 Fälle von Nephropathia gravidarum zeigten normale Verhältnisse.

III. 2 Fälle von Ikterus in der Gravidität zeigten Verzögerung in der Speicherung der infundierten Dextrose.

IV. 1 Fall von Chorea gravidarum und 1 Fall von Osteopathie (nicht Osteomalacie) in graviditate zeigten ebenfalls Verzögerung im Speicherungsvermögen der Leber.

Dex Harnzucker war in der Mehrzahl der Fälle negativ; indessen in einigen Fällen, bei zwei Wöchnerinnen, bei einer Nephropathia gravidarum und einem Fall von Osteopathie in graviditate als positiv befunden.

3. Eklampsien (vgl. S. 70 über Hyperglykämie sub partu): Die Eklampsien zeigten gegenüber Infusion einer Traubenzucker- oder Lävuloselösung ein recht typisches Verhalten. Es wurden Untersuchungen am Tage des Partus und im Wochenbett ausgeführt, die mit großer Regelmäßigkeit die gleichen Resultate zu ergaben. Am Tage des Partus besteht bei Eklampsien eine regelmäßige typische Störung im Speicherungsvermögen der Leber für Zucker. Der schon an und für sich erhöhte Blutzuckerspiegel steigt nach der Infusion erneut an. Eine Stunde nach der Infusion finden wir statt des Spiegels vor der Infusion einen wesentlich höheren Spiegel, der erst im Verlaufe von mehreren Stunden zum Ausgangswert zurücksinkt. Man darf nicht außer acht lassen, daß der schon an und für sich erhöhte Spiegel sub partu (s. oben S. 70) ein Ausdruck der Leberinsuffizienz darstellt. Zu dem addiert sich nun noch die Belastung durch die In- 
fusion. Was der normalen Leber spielend gelingt, vermag die eklamptische Leber nicht zu leisten. Die Speicherung des infundierten Zuckers innerhalb einer Stunde mißlingt. Die Blutzuckerwerte bleiben längere Zeit als in der Norm erhöht. Als Beispiele mögen einige kurze Zahlenangaben dienen:

$\begin{array}{cc}\text { Nüchternwert } & 1 \text { std. nach Infusion } \\ 0,165 & 0,195 \\ 0,080 & 0,161 \\ 0,107 & 0,153 \\ 0,099 & 0,112\end{array}$

Ausführlichere Angaben finden sich auf S. 80 u. 81, Tab. IIb. Auch im Anfang des Wochenbettes treffen wir in den ersten Tagen post partum hochgradige Verzögerungen im Vermögen, den infundierten Trauben. zucker zu speichern.

Das Speicherungsvermögen der Leber im Wochenbett kehrt ähnlich dem Sinken des Nüchternblutzuckerspiegels bald rascher, bald langsamer wieder zur Norm zurück.

Was den Harnzucker anbelangt, so war er auch hier bei diesen großen Blutzuckersteigerungen frei von Zucker, dagegen verschiedene Male im Wochenbett bei niedrigem Spiegel und Lävuloseinfusionen positiv, was wohl auf einen renalen Ursprung dieser Glykosurien hindeutet.

Der Einflub einer Injektion von $0,3 \mathrm{ccm}$ oder $0,75 \mathrm{ccm}$. Adrenalin auf den Blutzuckerspiegel.

Blum entdeckte als erster, daß eine Injektion von Adrenalin subcutan zu Glykosurie führt. Neuere Arbeiten stellten dann fest, daß es beim gesunden Menschen nur dann zur Glykosurie kommt, wenn mit dem Adrenalin zusammen Kohlenhydrate verfüttert werden (Landau).

Indessen tritt nach einer Injektion von $0,75 \mathrm{ccm}$ Adrenalin fast immer eine Hyperglykämie auf. Der ins Blut ausgeschwemmte Zucker wird durch Adrenalin in der Leber mobilisiert. Der Leber fällt durch die experimentelle Adrenalin-Hyperglykämie eine Mehrarbeit zu; eine weitere Mehrarbeit liegt in der Rückspeicherung des ausgeschwemmten Zuckers. Wir sehen im Ablauf dieser Blutzuckerwelle eine Belastung der Leber, die evtl. als „Funktionsprüfung der Leber“ Aufschluß über ihren Zustand geben könnte.

Ryser stellte auch schon Untersuchungen an, in denen er nachwies, daß Darreichungen wirksamer Substanzen aus Blutdrüsen keinen wesentlichen Einfluß auf den Blutzuckerspiegel bei Gravidae und nicht graviden Frauen ausüben. Indessen fand er bei Injektion von $0,1 \mathrm{ccm}$ Adrenalin bei Schwangeren oft Glykosurie, die er als renalen Ursprungs ansieht, und deren Eintreten er als Zeichen einer Nierenschädigung auffaßt. 
sub partu, im Wochenbett und bei Eklampsie.

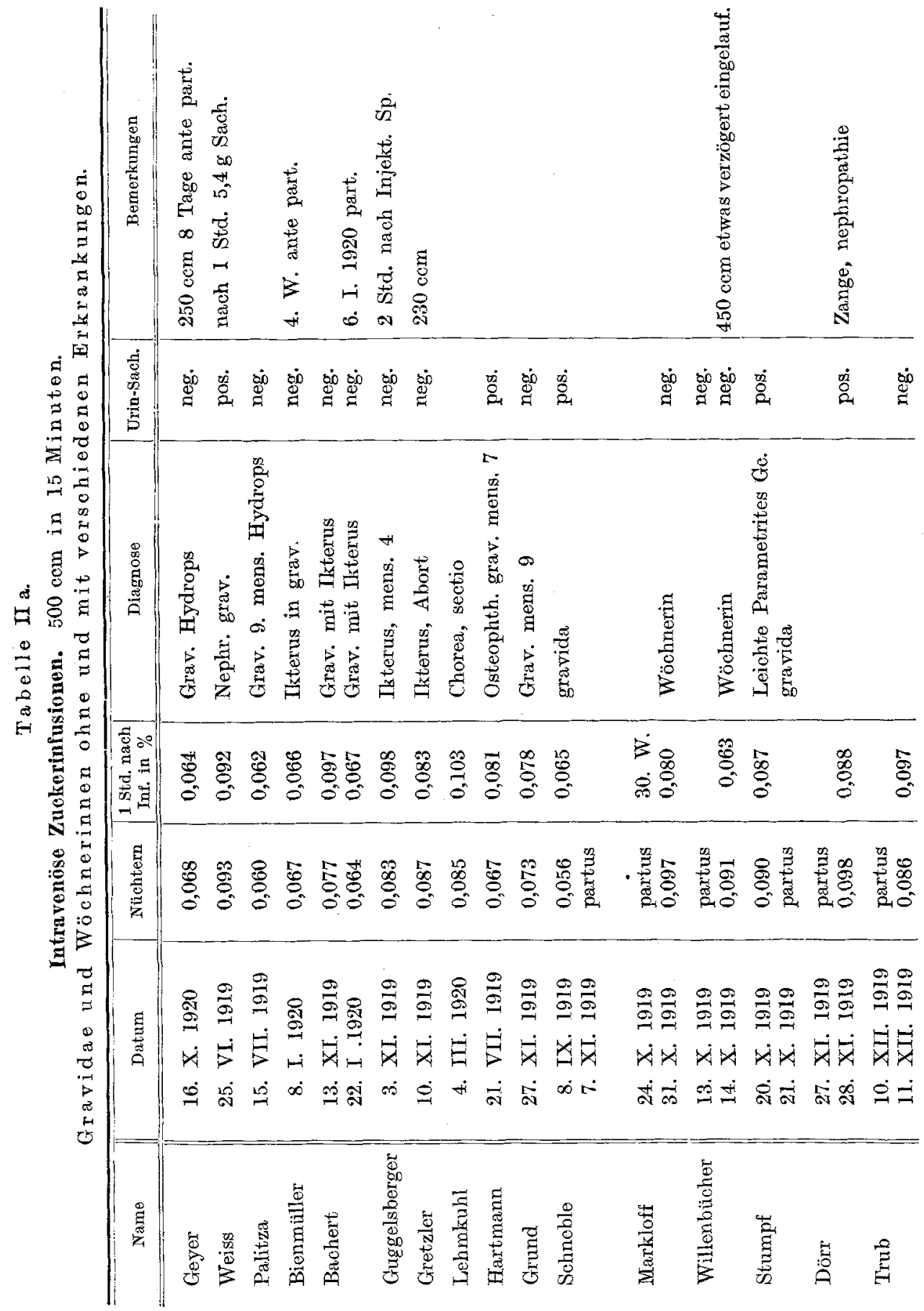




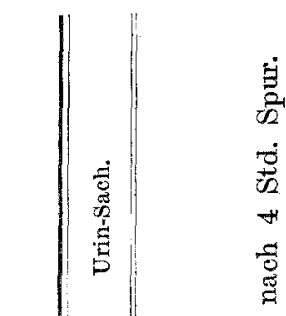

官

$\dot{\nabla}$

要

अ

Lo

ᄅ료

$\stackrel{5}{\stackrel{3}{0}}$

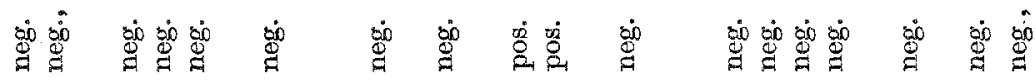

圖

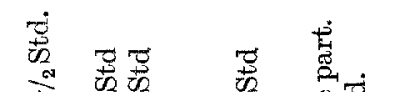

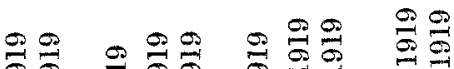

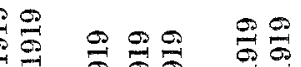

욤 $\stackrel{\sigma}{\sigma} \sigma \stackrel{\sigma}{\sigma} \stackrel{\sigma}{\sigma}$

봉

.

ลิ่

का

时

$\stackrel{\theta}{\sigma} \stackrel{\sigma}{\sigma}$

$\frac{\sigma}{\sigma} \sigma$

$\stackrel{20}{*} 0$

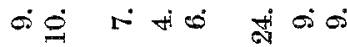
जi

งิ่

मं

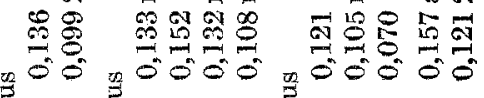
焉<smiles>[CH][CH]</smiles> 

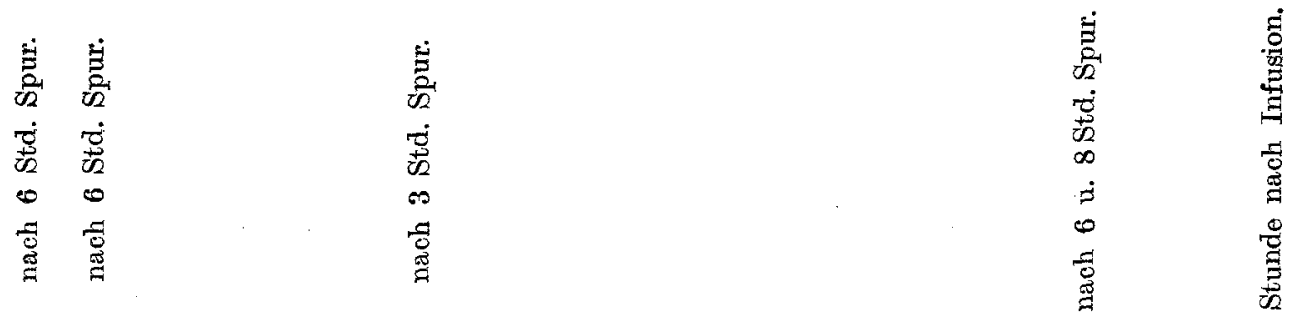

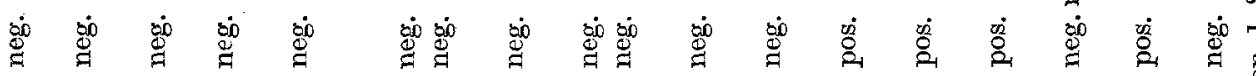

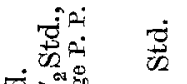

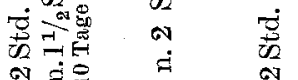

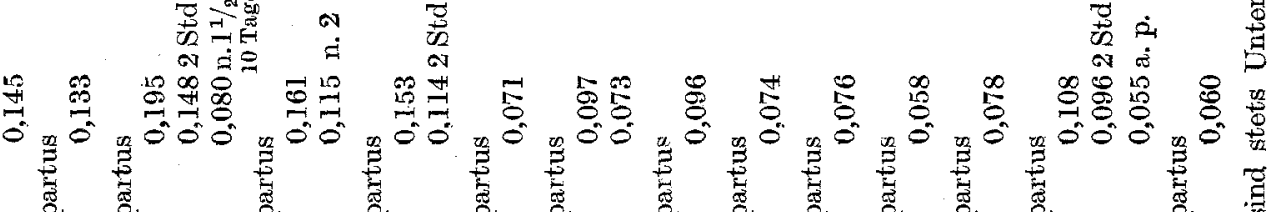

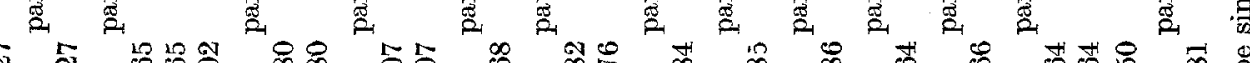

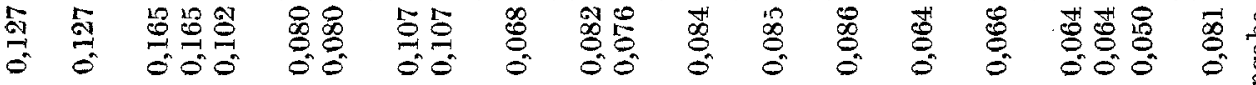

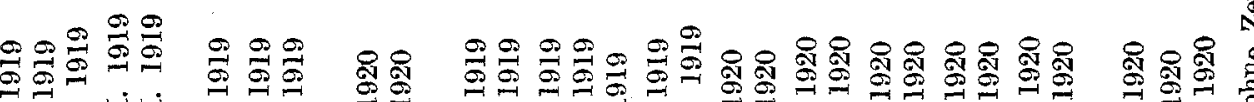
味 Nஸ்

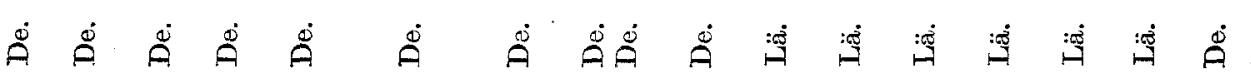

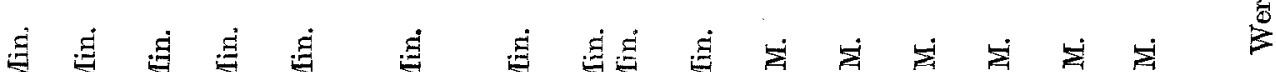

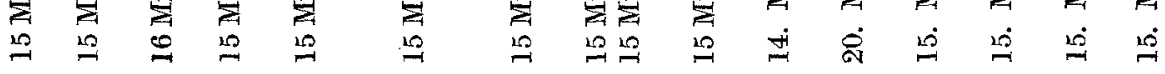

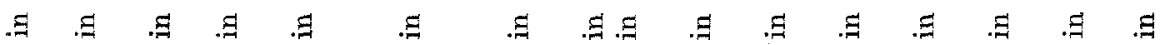

ㅇํำ

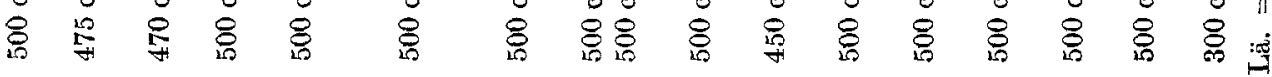

望 Archiv f. Gynäkologie. Bd. 116. 


\section{Eigene Untersuchungen.}

Fs lag uns bei unseren. Untersuchungen daran, die Befunde R ysers auf ihre Richtigkeit nachzuprüfen und ferner den Einfluß des Adrenalins auf die Eklampsieleber zu studieren.

Die Untersuchungen wurden in der Weise ausgeführt, daß nüchtern 0,3, meistens aber 0,75 Adrenalin subcutan injiziert wurde. Vor der Injektion wurde der Blutzuckerspiegel nach Bang bestimmt. Es wurden dann nach 20, 30, 40, 50 Minuten, einer Stunde und 2 Stunden nach der Injektion Prüfungen des Blutzuckerspiegels vorgenommen. In dieser Weise wurde der Einfluß der Adrenalininjektion auf das Zuckermobilisationsvermögen der Leber verfolgt. Ebenso wurde der Harnzucker geprüft und der Blutdruck registriert. Um einen möglichst objektiven Vergleich zu haben, zogen wir Lebergesunde, quasi normale Fälle, zu, die uns als Vergleichswerte dienen sollten.

So teilen sich unsere Fälle in:

1. Normale Fälle;

2. Normale Gravidae und Wöchnerinnen;

3. Eklampsien.

1. Normale Fälle. Es standen uns 12 normale Fälle, 1 Blutung, 1 Prolaps, 2 Dysmenorrhöen, 1 nervöse Erschöpfung und 7 Psychoneurosen zur Verfügung. $\mathrm{Zu}$ den Psychoneurosen ist zu bemerken, daß sie meist nur eine Dosis von $0,3 \mathrm{ccm}$ Adrenalin, also eine unterschwellige Dosis erhalten haben.

Die besten Vergleichswerte bilden in der Tat die 7 ersten Fälle, die eine Dosis von $0,75 \mathrm{ccm}$ Adrenalin verabreicht bekamen.

Die Blutzuckerwerte vor der Injektion sind durchwegs normal. Durchschnitt $0,083 \%$. Die Injektion hat regelmäBig ein Ansteigen des Blutzuckerspiegels zur Folge. Es wird ein Höhepunkt der Ausschüttung erreicht und dann fällt der Spiegel wieder zur Norm zurück. Die Injektion bewirkt bei verschiedenen Personen eine recht verschiedene Stärke der Zuckerausschüttung, eine Verschiedenheit der Zeit, in der der Höhepunkt der Ausschüttung erreicht wird, und eine Verschiedenheit des Andauerns des erhöhten Blutzuckerspiegels.

Um einen Einblick in den Sinn dieser Zahlen zu bekommen, versuchten wir folgendermaßen vorzugehen:

Wir bestimmten den zahlenmëßigen Unterschied zwischen dem Wert vor der Injektion und allen Werten nach der Injektion.

Beispiele:

Wert pro $100 \mathrm{ccm}$ Blut vor der Injektion von $0,75 \mathrm{ccm}$ Adrenalin: 93 .

Werte pro $100 \mathrm{~cm}$ Blut nach der Injektion von $0,75 \mathrm{~cm}$ Adrenalin :

Nach $20 \mathrm{Min}$.

120 Differenz
$\mathbf{2 7}$
Nach $40 \mathrm{Min}$. 105
Differenz

12 
Die Differenzwerte geben an, um wieviel der Blutzuckerspiegel gestiegen ist. Führt man diese Rechnung aus, insofern die Patienten 0,75 Adrenalin erhalten haben, addiert dann die einzelnen Werte in 20, in 30 usw. Minuten und teilt die Summe der Werte nach 20, nach 30 Minuten usw. durch die Anzahl der Untersuchungen, so erhält man für 20 Minuten, für 30 Minuten usw. nach der Infusion einen Durchschnittswert der Steigerung des Blutzuckerspiegels nach Adrenalininjektion.

Diese Berechnung ergibt nun bei normalen Fällen:

In 20 Minuten nach Injektion um 21,2 gestiegen

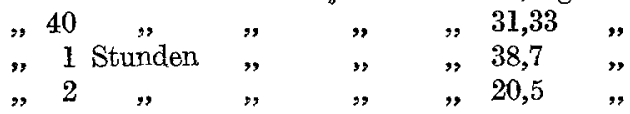

Man sieht, daB die Wirkung des Adrenalins in einer Stunde nach der Injektion ihren Höhepunkt erreicht und dann in ihrer Wirkung nachläßt.

Die letzten 5 Psychoneurosen sind weniger zum zahlenmäßigen Vergleich heranzuziehen, weil sie nur die Dosis von 0,3 Adrenalin erhielten. Indessen zeigen sie doch auch deutlich eine Hyperglykämie als Reaktion auf die Einverleibung des Adrenalins. Die Werte sind 50 Minuten und 1 Stunde nach der Injektion gemessen worden.

Der Blutdruck ist schwankend, ohne indessen höhere Werte als $134 \mathrm{~mm}$ zu erreichen.

Auch die Untersuchung auf Harnzucker fällt negativ aus, außer in 2 Fällen, wo Spuren auftreten, was Landaus Ansicht entspricht, daß eine Adrenalininjektion allein noch nicht genügt, um beim Gesunden Glykosurie hervorzurufen.

2. Normale Gravidae und Wöchnerinnen: Normale Gravidae und Wöchnerinnen zeigen auf die Injektion von $0,75 \mathrm{ccm}$ Adrenalin hin keine besonderen Unterschiede im Vergleich zu Nichtgraviden, Ryser. Dies mag für die Gravidität im allgemeinen und für das Wochenbett recht gut stimmen. Indessen ließen sich kurz ante und post partum recht deutliche Abweichungen von diesen Befunden erheben.

Schon der Fall (Lippert), obschon 3 Wochen ante partum, zeigt gegenüber anderen Fällen von Tab. III a eine verminderte Reaktionsfähigkeit der Leber auf Adrenalin. Auch hier habe ich wieder die zahlenmäßigen Unterschiede zwischen Nüchternwerten und den verschiedenen Werten zu verschiedenen Zeiten nach Injektion als Vergleichsmaß herangezogen. So ergibt dieser Fall verminderte Vergleichswerte gegenüber der Norm von 21 nach 50 Minuten, 15 nach 1 Stunde und 8 nach 2 Stunden (s. oben).

Ich stellte nun die Vergleichswerte von 4 Fällen im Zeitraum vom 5. bis 1. Tag ante partum, und 1. bis 7. Tag post partum zusammen, 
um die Reaktion der Leber auf Adrenalinreizung unmittelbar vor und nach der Geburt zu untersuchen.

Durchschnittswert ante partum 9,5
20,5
8,75
13,25
Durchschnittswert post partum 22,5 20 Min. nach Injektion $31,75 \quad 40$ 25,751 Stde., $12,52 "$,

Aus den durchschnittlichen Vergleichswerten geht mit aller Deutlichkeit hervor: Sowohl kurz ante wie post partum besteht ein vermindertes Ansprechen der Leber auf Adrenalinreizung; ferner spricht kurz ante partum die Leber im Vergleich zu kurz post partum in geringerem Maße auf Reizung an. Wie verhält sich nun die Leber sub partu und in den ersten Stunden post partum? Die Werte sind ganz typisch, so daß ich glaube, hier etwas Charakteristisches zu sehen.

Tabelle III a. Blutzuckergehalt nach Injektion von Adrenalin in mg per $100 \mathrm{ccm}$. Normale Fälle.

\begin{tabular}{|c|c|c|c|c|c|c|c|c|c|c|c|}
\hline Name & Datum & Adr. & Vor & $\begin{array}{c}\text { Maci } \\
20 \\
\text { Min }\end{array}$ & $\begin{array}{c}\text { Nach } \\
30 \\
\text { Miiv. }\end{array}$ & 40 & $\mid \begin{array}{c}\text { Macb } \\
50 \\
\text { Min. }\end{array}$ & $\left|\begin{array}{c}\mathrm{Nach} \\
1 \\
\text { std. }\end{array}\right|$ & \begin{tabular}{|c|c|} 
Tiach \\
$\mathbf{2}$ \\
sta.
\end{tabular} & Urin-Sach. & Blutdruck \\
\hline Brieger & $\begin{array}{l}\text { 15. IV. } 1919 \\
\text { 16. IV. } 1919\end{array}$ & $\begin{array}{l}0,75 \\
0,3\end{array}$ & 94 & $\begin{array}{l}114 \\
114\end{array}$ & & $\begin{array}{l}161 \\
127\end{array}$ & & 133 & 104 & $\begin{array}{l}\text { neg. } \\
\text { neg. }\end{array}$ & $\begin{array}{l}90,120,135,130,120 \\
108,100\end{array}$ \\
\hline Bock & 30. IV. 1919 & 0,75 & 93 & 105 & & 114 & & 106 & 126 & neg. & $130,120,115,120,120$ \\
\hline Christ & 11. VII. 1919 & 0,75 & 88 & & 113 & 116 & 142 & 134 & 101 & neg. & $110,120,110,105$ \\
\hline Zeh & 4. XII. 1919 & $0,75 \mid$ & 86 & 115 & & 121 & & 141 & 95 & n. 8 st. Sp. & $110,110,110,115,115$ \\
\hline Menger & 2. ПТ. 1920 & 0,75 & 94 & 98 & & 101 & & 123 & 123 & neg. & $100,105,100,110,105$ \\
\hline Willenecker & 9. XПТ, 1919 & 0,75 & 70 & 111 & & 100 & & 105 & 199 & neg. & $110,120,110,100$ \\
\hline Faller & $\begin{array}{ll}\text { 2. XII. } 1919 \\
\text { 8. XII. } 1919\end{array}$ & $\begin{array}{l}0,3 \\
0,75\end{array}$ & $\begin{array}{l}71 \\
71\end{array}$ & & & & $\begin{array}{r}87 \\
123\end{array}$ & $\mid \begin{array}{r}84 \\
125\end{array}$ & & $\begin{array}{l}\text { neg. } \\
\text { neg. }\end{array}$ & $\begin{array}{l}130 \\
130\end{array}$ \\
\hline Pohl & 27. I. 1920 & 0,3 & 73 & & & & 97 & 95 & & neg. & 110 \\
\hline Hübner & 26. I. 1920 & 0,3 & 82 & & & & 91 & 87 & & neg. & 110 \\
\hline Kriegsbaum & 20. XI. 1919 & 0,3 & 76 & & & & & 105 & & n. 1 St. Sp. & 110 \\
\hline Birkenstein & 28. Х. 1919 & 0,3 & 76 & & & & 88 & 71 & & & 80 Vor I. \\
\hline Schmitt & $\mid \begin{array}{lll}\text { 17. } & \text { XI. } & 1919 \\
\text { 29. } & \text { XI. } & 1919\end{array}$ & $\begin{array}{l}0,3 \\
0,3\end{array}$ & $\begin{array}{l}90 \\
79\end{array}$ & & & & $\begin{array}{r}97 \\
106\end{array}$ & $\begin{array}{l}102 \\
119\end{array}$ & & $\begin{array}{l}\text { neg. } \\
\text { neg. }\end{array}$ & $\begin{array}{l}120 \\
120\end{array}$ \\
\hline
\end{tabular}

50 Minuten nach der Injektion von $0,75 \mathrm{ccm}$ Adrenalin ist im Durchschnitt unserer Fälle der Blutzuckerspiegel gegenüber dem Nüchternwert vor der Injektion $1 \mathrm{~m}$ 0,5 gesunken, nach einer Stunde erhebt er sich um 11,5, um nach 2 Stunden um 1 zu fallen. Wir finden also sub partu und in den Stunden post partum ein völliges Versagen der Leber, auf eine Reizung von Adrenalin hin mit Zuckerausschwemmung ins Blut zu antworten; ja im Gegenteil, der Blutzuckerspiegel sinkt.

Der Blutdruck zeigt wechselnde Höhen bei verschiedenen Patienten. Teils hält er sich in normalen Grenzen, teils steigt er nach Injektion bis zu Werten oberhalb des Normalen. 
Der Harnzucker ist nach. Injektion von $0,75 \mathrm{ccm}$ Adrenalin, wie $R y$ ser schon feststellte, in vielen Fällen positiv.

3. Eklampsien: Die Eklampsiewöchnerinnen reagieren nicht regelmäßig in charakteristischer Weise auf eine Injektion von $0,75 \mathrm{ccm}$ Adrenalin. Die Durchschnittswerte für $0,75 \mathrm{ccm}$ Adrenalin halten

Tabelle IIIb. Gravidae und Wöchnerinnen.

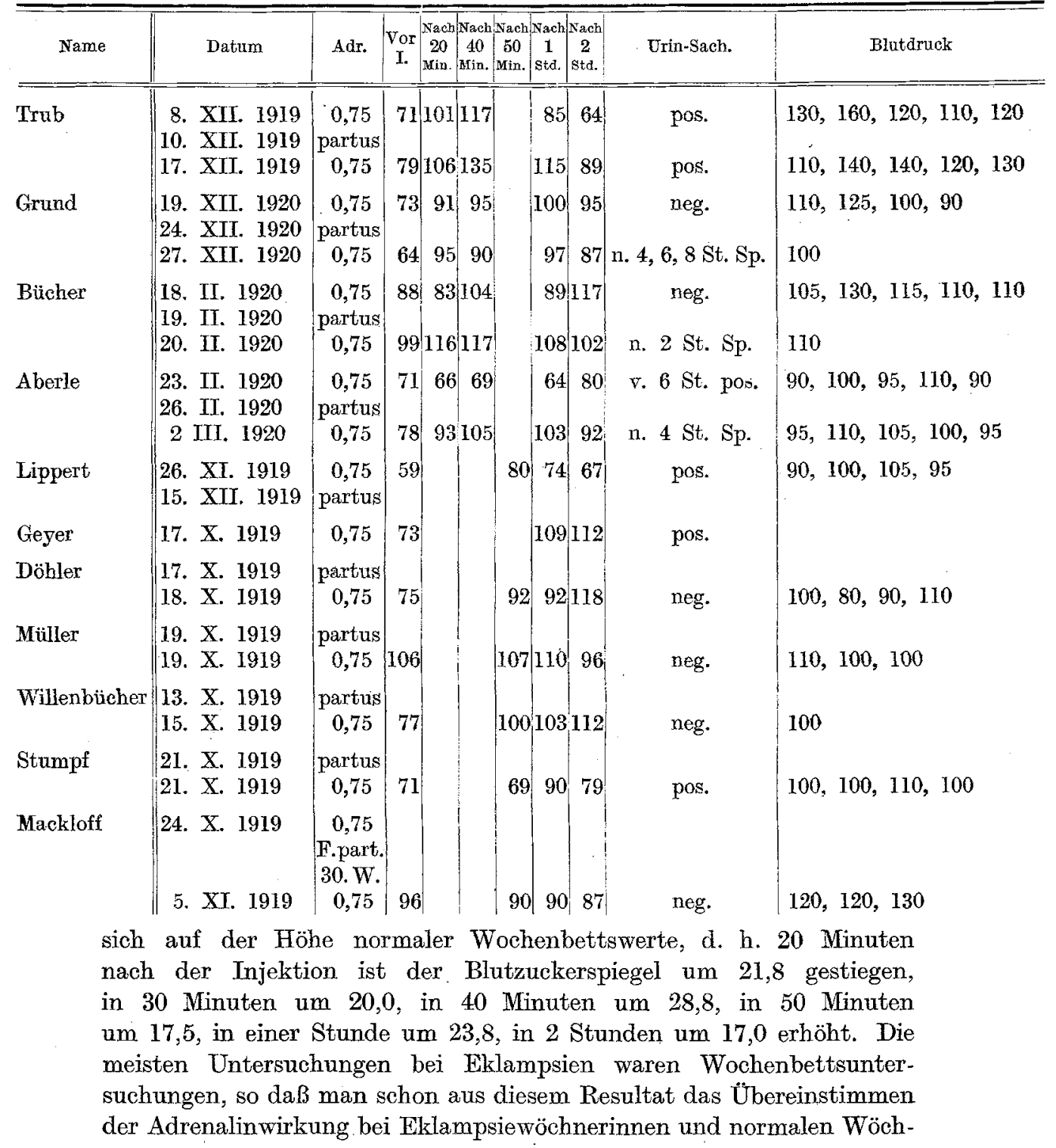


Tabelle III c. Eklampsien.

\begin{tabular}{|c|c|c|c|c|c|c|c|c|c|c|c|}
\hline Name & Datun & Adr. & Vor & $\begin{array}{c}\text { Nach } \\
20 \\
\text { Min. }\end{array}$ & 30 & $\mid \begin{array}{c}\mathrm{Nach} \\
10 \\
\mathrm{Min}\end{array}$ & $\begin{array}{c}\mathrm{Nach} \\
50 \\
\text { Min }\end{array}$ & $\mid \begin{array}{c}1 \\
1 \\
\text { std. }\end{array}$ & $\begin{array}{c}\text { Nach } \\
2 \\
\text { istd. }\end{array}$ & Urin-Sach. & Blutdruck \\
\hline Lautz & $\begin{array}{c}\text { 20. VIII. } 1919 \\
25 . \text { VIII. } 1919 \\
\text { 2. IX. } 1919\end{array}$ & $\begin{array}{c}\text { partus } \\
0,75 \\
0,75\end{array}$ & $\begin{array}{r}106 \\
70\end{array}$ & & & & $\left|\begin{array}{r}141 \\
89\end{array}\right|$ & $\begin{array}{r}1531 \\
95\end{array}$ & $\begin{array}{r}144 \\
94\end{array}$ & $\begin{array}{l}\text { pos, } \\
\text { neg, }\end{array}$ & $\begin{array}{l}125,130,140,130 \\
110,120,120,120\end{array}$ \\
\hline Diener & $\begin{array}{lll}11 . & \text { II. } 1920 \\
13 . & \text { II. } & 1920\end{array}$ & $\begin{array}{c}\text { sectio } \\
0,75\end{array}$ & 73 & 89 & & 105 & & 1041 & 115 & n. 6 St. Sp. & $120,120,130,100$ \\
\hline Gretschel & $\begin{aligned} \text { 9. } & \text { IX. } 1919 \\
12 . & \text { IX. } 1919 \\
\text { 9. } & \text { X. } 1919\end{aligned}$ & $\begin{array}{c}\text { partus } \\
0,75 \\
0,75\end{array}$ & $\begin{array}{l}97 \\
94\end{array}$ & & & & $\begin{array}{r}86 \\
135\end{array}$ & $\begin{array}{r}82 \\
122\end{array}$ & $\begin{array}{l}52 \\
76\end{array}$ & $\begin{array}{l}\text { neg. } \\
\text { neg. ? }\end{array}$ & $\begin{array}{l}130,140,140 \\
100,115,100\end{array}$ \\
\hline Zimmer & $\begin{array}{rlr}\text { 4. } & \text { XI. } & 1919 \\
\text { 5. XI. } & 1919 \\
\text { 25. XI. } & 1919\end{array}$ & $\begin{array}{c}\text { partus } \\
0,75 \\
0,75\end{array}$ & $\begin{array}{l}73 \\
94\end{array}$ & & & & 135 & $\begin{array}{l}100 \\
125\end{array}$ & 108 & $\begin{array}{l}\text { neg. } \\
\text { Sp. }\end{array}$ & $\begin{array}{l}110,120 \\
100,120,110 \\
100\end{array}$ \\
\hline Schäfer & $\begin{array}{ll}\text { 19. III. } 1920 \\
\text { 26. III. } 1920\end{array}$ & $\begin{array}{c}\text { sectio } \\
0,75\end{array}$ & 62 & 82 & & 94 & & 99 & 94 & neg. & $\begin{array}{l}100,105,110 \\
105,115\end{array}$ \\
\hline Swoboda & $\begin{array}{l}\text { 11. IV. } 1920 \\
\text { I4. IV. } 1920\end{array}$ & $\begin{array}{c}\text { entbundee } \\
\text { eingeliefert } \\
0,75\end{array}$ & 67 & & 97 & & & 104 & 98 & neg. & $120,135,140$ \\
\hline Heeb & $\begin{array}{ll}22 . & \text { IV. } 1920 \\
26 . & \text { IV. } 1920\end{array}$ & $\begin{array}{c}\text { partus } \\
0,3\end{array}$ & 68 & 66 & & 90 & & 84 & 88 & pos.? & $150,145,150$ \\
\hline Weiler & $\begin{aligned} 29 . & \text { III. } 1920 \\
3 . & \text { IV. } 1920\end{aligned}$ & $\begin{array}{l}\text { sectio } \\
0,3\end{array}$ & & 130 & & 114 & & 95 & 99 & neg. ? & $165,160,160,155$ \\
\hline Heuser & $\begin{array}{lll}\text { 12. } & \text { V. } 1920 \\
31 . & \text { V. } & 1920\end{array}$ & $\begin{array}{l}0,3 \\
\text { F. partus } \\
36 . W .\end{array}$ & 66 & 79 & & 62 & $\mid$ & 67 & 80 & neg. & $\begin{array}{l}175160,170,170 \\
170\end{array}$ \\
\hline Kirchner & $\begin{aligned} \text { 9. } & \text { XIT. } 1919 \\
10 . & \text { XII. } 1919\end{aligned}$ & $\begin{array}{c}\text { partus } \\
0,75\end{array}$ & & 120 & & 133 & & 1281 & 123 & neg. & $110,100,95$ \\
\hline Streitenberger & $\mid \begin{array}{ll}25 . & \text { VIII. } 1919 \\
15 . & \text { IX. } 1919\end{array}$ & $\begin{array}{c}\text { partus } \\
0,75\end{array}$ & 72 & & & & 69 & 66 & 82 & neg. & $130,140,140$ \\
\hline Kümpel & $\mid \begin{array}{lll}\text { 12. } & \text { XTI. } 1919 \\
\text { 12. } & \text { XII. } 1919\end{array}$ & $\begin{array}{l}\text { partus } \\
0,75\end{array}$ & 114 & & 124 & & & 1151 & 116 & neg. & 135 \\
\hline Käkel & $\begin{array}{l}24 . \text { II. } 1920 \\
\text { 13. III. } 1920\end{array}$ & $\begin{array}{c}\text { sectio, } \\
\text { F. part. } \\
\text { 38. W. } \\
0,75\end{array}$ & & 100 & & 103 & & 114 & 80 & neg. & $\begin{array}{l}120,140,120 \\
110,115\end{array}$ \\
\hline Rein & $\begin{array}{l}\text { 11. IX. } 1919 \\
\text { 13. IX. } 1919\end{array}$ & partus & 55 & & & & 79 & 83 & 85 & neg. & $\begin{array}{l}130,140,140 \\
150\end{array}$ \\
\hline Baschnagel & $\mid \begin{array}{lll}20 . & \text { IX. } & 1919 \\
25 . & \text { IX. } & 1919 \\
\text { 9. } & \text { X. } & 1919\end{array}$ & $\begin{array}{c}\text { partus } \\
0,75 \\
0,75\end{array}$ & $\begin{array}{l}88 \\
83\end{array}$ & & & & $\begin{array}{l}87 \\
96\end{array}$ & $\begin{array}{r}93 \\
118\end{array}$ & $\begin{array}{r}99 \\
121\end{array}$ & $\begin{array}{l}\text { neg. } \\
\text { neg. }\end{array}$ & \\
\hline Funk & $\begin{array}{lll}28 . & \text { XI. } & 1919 \\
\text { 29. } & \text { XI. } & 1919\end{array}$ & $\begin{array}{c}\text { partus } \\
0,75\end{array}$ & 76 & 95 & & 86 & & 93 & 81 & neg. & $\begin{array}{l}140,160,145 \\
140,130\end{array}$ \\
\hline
\end{tabular}


nerinnen erkennen kann. Ante partum wurde nur eine einzige Bestimmung bei einem Eklampsieanfall gemacht. Sie zeigt ungefähr 2 Wochen ante partum ausgeführt, ein geringes Ansprechen der Leber auf Adrenalin. Ein einziger Wert $s u b$ partu zeigt wie bei normalen Parturientes ein schlechtes Ansprechen der Leber auf die Adrenalinreizung hin.

Betrachtet man einzelne Fälle von Wöchnerinnen, so erkennt man, daß die verschiedenen Patienten durchaus verschiedene Peaktionen auf die Adrenalininjektion hin zeigen. Die einen reagieren mit sehr starker Zuckerausschüttung (Lautz, Schäfer), die meisten normal bis subnormal, bei einigen fällt auf die Injektionen hin der Blutzuckerspiegel unter den Wert vor Injektion (Gretschel, Streitenberger). Auch ist in vielen Fällen der Zweistundenwert der höchste, so daß wir hier eine verzögerte Antwort der Leber auf Adrenalinreizung vor uns haben (Diener, Zimmer, Heb, Weiler, Streitenberger), wie es auch bei normalen Wöchnerinnen gelegentlich beobachtet wird.

So bietet die Adrenalininjektion in ihrer Wirkung auf die Höhe des Blutzuckerspiegels ein mannigfaltiges Bild der Steigerungsmöglichkeiten dar. Es zeigen sich verschiedene Arten abnormer Reaktionen einzelner Fälle auf Adrenalininjektion hin, ohne indessen für die Eklampsie als Ganzes etwas Charakteristisches darzustellen.

Der Blutdruck ist teils schon vor, teils erst auf die Injektion von Adrenalin hin erhöht, was zum Bilde der Eklampsie gehört. Der Harn ist teils positiv, in den meisten Fällen bleibt er jedoch frei von Zucker.

\section{Der Urobilingehalt des Urins in Graviditate, sub partu und bei Wöeh- nerinnen und Eklamptischen.}

Urobilinurie ist das feinste Zeichen der Leberschädigung, Fischler. Urobilin ist der einzige Leberfarbstoff, dessen Auftreten im Urin wirklich pathognomonisch für eine vorhandene Leberschädigung sein kann. Tritt Urobilin im Urin auf, so ist dies immer ein Zeichen der Insuffizienz der Leber, und zwar in dem Sinne, daß das aus dem Darme ins Blut rückresorbierte Urobilin von der Leber nicht gespeichert werden kann und durch die Niere ausgeschieden wird. Nun sind aber so viele Krankheiten mit einer so leichten Leberschädigung einhergehend, daß ein rein qualitativer Nachweis des Urobilins zu keinen Vergleichswerten führt. Schlesinger hat nun mit der Zinkacetat-AlkoholFluorescenzprobe eine quantitative Methode geschaffen, die es erlaubt, $0,002 \%$ mit Sicherheit nachzuweisen. Nicht zu vergessen bei allen Untersuchungen sind die Beeinflussungen der Werte durch die eingenommene Nahrung. Es gibt eine physiologische Urobilinurie, die schwankt zwischen $20 \mathrm{mg}$, Müller, und 80-140 mg, Hop pe - Se yler, pro Tagesmenge. Diese extremsten Differenzen der verschiedenen 
Autoren sind nur erklärlich durch den Einfluß der Nahrung, wie er besonders von Grimm in Versuchen an sich selbst festgestellt wurde. Eigentliche Vergleichswerte sind also nur Nüchternwerte. Im nüchternen Zustand soll der Urin kein Urobilin oder nur Spuren enthalten.

Auch für die Schwangerschaft, die eine vermehrte Leberinanspruchnahme bedingt, wurde der Urobilingehalt des Urins nachgeprüft. Mer letti fand in den letzten Monaten der Schwangerschaft eine Vermehrung des Urobilins, Seitz konnte die Angaben bestätigen. Fr fand in $60 \%$ sämtlicher Schwangeren die Urobilinprobe positiv gegen $8 \%$ bei Nichtschwangeren.

\section{Eigene Untersuchungen.}

Unsere Resultate erhielten wir auf zwei Arten. Die älteren Resultate stellen rohe Schätzungen dar, die mit + oder positiv, ++ und +++ charakterisiert wurden. Als Methode diente die Schlesingersche Zinkacetat-Alkoholprobe. Später, als wir im Besitze einer Urobilinskala waren, konnten wir genauere Studien über den Prozentgehalt und die Milligrammurobilin anstellen. Die Milligrammurobilin wurden so bestimmt, daß der $\frac{\text { Prozentgehalt mal Menge }}{100}$ des Urins die Milligrammzahl ergab. Um alte und neve Bestimmungen einigermaßen unter einem Gesichtspunkte verwerten zu können, wurde durch Vergleich der: neuen Methode mit den alten Schätzungen festgestellt, daß

$$
\begin{array}{ccccc}
+ \text { oder Pos. einem Werte von } & 0,002-0,01 \% \\
++ & " & \Rightarrow & 0,01-0,024 \% \\
+++ & " & , & & 0,024 \text { und mehr } \%
\end{array}
$$

entspricht. Wegen Platzmangels und wegen des negativen Ergebnisses dieser Untersuchungen wurden die Tabellen weggelassen.

Es liegen nun folgende Untersuchungen vor:

1. Der Urobilingehalt bei 12 Stunden Urin;

2. Der Einfluß einer Adrenalininjektion auf den Urobilingehalt des Urins.

1. Der Urobilingehalt in Sammelurinen von 12 Stunden. Es wurden vorerst einige normale Nichtgravidae untersucht, um einen Vergleichswert mit den späteren Werten zu erhalten. Der in verschiedenen Portionen gesammelte und geprüfte Urin zeigt entweder kein oder nur Spuren von Urobilin. Da, wo wir höhere Werte bekommen, spielt stets das Essen die ausschlaggebende Rolle. Es ist dies überhaupt der Nachteil der 12-Stundenurine, daß die Nahrung als Faktor nicht ausgeschaltet ist. Was wohl recht typisch für normale Leberfunktion ist, ist, daß der Nullwert, der erste Wert für den endgültigen Zwölfstundenwert, stets frei von Urobilin ist. Die Untersuchungen wurden nüchtern begonnen, so daß dieser negative Nüchternwert einen Ausdruck der 
normalen Leberfunktion darstellt. Man kann direkt aus den einzelnen Urinportionen ersehen, ob die Patientin gegessen hat oder nicht. Solange sie nüchtern war, hält sich der Urobilinspiegel in den untersten Grenzen oder ist negativ. Sobald die Patientin ißt, steigen Harnmengen, Prozentgehalt und Milligrammurobilin an. Die Summe von Milligrammen in 12 Stunden ist äußerst wechselnd und stellt eine Variante der Art und Menge der eingenommenen Speisen dar. Berechnungen der verschiedenen Portionen in 12 Stunden auf einen Durchschnittswert ergeben im allgemeinen nur geringste Spuren von Urobilin im Prozentgehalt.

Gravidae und Wöchnerinnen: Es wurde nur von vier $\mathrm{Pa}$ tientinnen der Sammelurin bestimmt. Das einzige, was zum Unterschiede von Nichtschwangeren auffällt, ist, daß in 2 Fällen der Nullwert, also Nüchternwert, einen positiven Urobilingehalt aufweist.

Eklampsien: Auch hier zeigt der Urobilingehalt des Zwölfstundenurins keine Besonderheiten. Die Null- oder Nüchternwerte sind in den fraktioniert untersuchten Fällen außer in 2 Fällen negativ. Weder vor oder nach dem Partus sind Besonderheiten zu bemerken. Kurz post partum ist sogar der Urobilingehalt, auf 12 Stunden berechnet, meist negativ oder nur in Spuren vorhanden, was mit der verminderten Nahrungsaufnahme in den ersten Tagen post partum zusammenhängt. Einige Tage später tritt der Einfluß der Nahrung wieder zur Geltung, es treten höhere Werte auf.

Der Zwölfstundensammelurin bietet somit kein gutes Mittel für Untersuchung auf eine pathologische Urobilinurie und zeigt durch den Einfluß der Nahrung zu große Schwankungen im Physiologischen, um Schlüsse auf pathologische Vorgänge ziehen zu können.

2. Der EinfluB einer Adrenalininjektion auf den Urobilingegehalt des Urins. Ausgehend von dem Gedanken, daß bei einer Belastung der Leber, bei vermehrter Lebertätigkeit vermehrte Galle abgesondert wird, daß durch eventuelles Versagen der Leber in der Rückresorption des vermehrten Urobilins ein Einblick in eine Leberfunktionsstörung gewonnen werden könnte, wurden diese Untersuchungen unternommen. Es wurde so untersucht, daß der Gehalt an Urobilin vor der Injektion von Adrenalin bestimmt wurde; dann 2 und 4 Stunden nach der Injektion. Bis 4 Stunden nach der Injektion blieben die Patienten nüchtern. 6 und 8 Stunden nach der Injektion wurde noch eine Bestimmung ausgeführt, die beide unter dem Einfluß von Nahrungszufuhr stehen und dadurch Kontrolluntersuchung für die Empfindlichkeit der Leber wie der Schlesingerschen Urobilinprobe darstellen. Als Vergleichswerte dienen wieder einige Nichtschwangere, lebergesunde Fälle.

Nor male Fälle: Die Werte vor der Injektion von Adrenalin sind in der Mehrzahl negativ oder zeigen nur Spuren entsprechend dem 
Urobilingehalt von lebergesunden Nichtschwangeren. Der Einfluß der Adrenalininjektion von 0,3 oder $0,75 \mathrm{ccm}$ läßt sich für den Urobilingehalt des Urins kaum erkennen. Entweder bleibt der Urin in den ersten 4 Stunden negativ oder es treten nur Spuren in den Grenzen des Physiologischen auf. 6 und 8 Stunden nach Injektion tritt der Einfluß des Essens deutlich zutage.

Gravidität und Wochenbett: Die Werte vor der Injektion sind hier in 11 von 17 Fällen positiv. Es zeigt dies zum Unterschiede von 4 positiv gegen 9 negativ bei Nichtgraviden eine starke Vermehrung der positiven Werte in der Schwangerschaft. Sieht man die einzelnen Werte an, so sind weit höhere darunter zu finden als bei Nichtschwangeren, z. B. $0,016 \%$.

Der Einfluß des Adrenalins 2 und 4 Stunden nach Injektion ist durchwegs gering. Den meisten Einfluß finden wir noch 4 Stunden nach Injektion, doch sind die Steigerungen so gering, daß man nicht von einem Versagen der Leber sprechen kann. Einzig 2 Fälle, bei denen der Nüchternwert bereits erhöht ist, zeigen deutlich vermehrtes Urobilin nach Adrenalininjektion. Die Werte nach 6 und 8 Stunden bieten nichts Charakteristisches. Weder sub partu, noch ante und post partum zeigen sich außer den beiden genannten Fällen Besonderheiten.

Eine Injektion von Adrenalin hat in der Gravidität, sub partu und im Wochenbett keinen Einfluß auf den Urobilingehalt des Urins. Die Leber ist imstande, die Mehrarbeit der Urobilinrückresorption zu leisten.

Eklampsien: Von 32 Werten vor der Injektion von $0,75 \mathrm{ccm}$ Adrenalin finden wir in 16 Fällen den Urin frei von Urobilin; in 16 Fällen dagegen fiel die Reaktion positiv aus. Von den positiven Werten sind in 4 Fällen Spuren von Urobilin $=0,002 \%$ nachweisbar, in 8 weiteren Fällen bis $0,01 \%$ und in 4 Fällen über $0,01 \%$ Urobilin.

Es ist auch hier die Zahl der vor Injektion positiven Werte höher als in der Norm. Die Fälle, bei denen der Wert vor Injektion negativ war, zeigen fast ausschließlich keinen Einfluß auf die Adrenalininjektion hin. Entweder bleiben die Werte negativ oder es treten doch nur kleine Werte auf. Einzig 1 Fall zeigt 4 Stunden nach Injektion mehr als $0,01 \%$ Urobilin.

Bei den 8 Werten mit bis $0,01 \%$ vor Injektion finden wir auch außer 2 Fällen nach 0,75 bzw. 0,5 Adrenalin nur wenig Einfluß 2 und 4 Stunden nach Injektion.

SchlieBlich die vier Werte, bei denen mehr als $0,01 \%$ schon vor Injektion im Urin war. Es dauert die Urobilinurie weiter, fast ohne aber einen Einfluß der Adrenalininjektion erkennen zu lassen. Hieraus ist ersichtlich, daß die Adrenalininjektion bei Eklamptischen auf den Urobilingehalt des Urins nicht anders einwirkt als auf gesunde Gravidae und Wöchnerinnen, d. h. sie hat keinen nennenswerten Einfluß. 
Die Adrenalininjektion verbunden mit der Urobilinprobe gibt also keinen Aufschluß über eine Leberfunktionsstörung in Graviditate sub partu, bei Wöchnerinnen und Eklamptischen.

Der Reststickstoffgehalt des Blutes in Graviditate, sub partu, im Wochenbett und bei Eklampsien.

Der Reststickstoffgehalt des Blutes schwankt zwischen $0,02-0,04 \%$. Bei erhöhtem Reststickstoffgehalt des Blutes kommen zwei Möglichkeiten der Schädigung in Betracht:

1. Es kann sich um eine Schädigung der Leber handeln, vermindertes Vermögen, das Eiweiß zu Harnstoff abzubauen, Erhöh ung derA minorestquote. Oder

2. es handelt sich um eine Schädigung der Niere, im Sinne der Retention von Reststickstoffprodukten, hauptsächliches Ansteigen der Harnstoffquote im Blute, Bang.

Da nun bei vielen Fällen in der Schwangerschaft Kreatin, Aminosäuren und Ammoniak in vermehrtem Maße im Urin auftreten (Handbuch Döderlein: Seitz), wäre also möglich, daß die Steigerung des Rest-N-Spiegels bei Graviditätserkrankungen hepatogener Ursache wäre. Indessen kann nur dann Sicherheit bestehen, wenn neben dem Rest- $\mathrm{N}$ noch die Harnstoffquote bestimmt wird und so ihr Anteil am Rest- $\mathrm{N}$ übersehen werden kann.

\section{Eigene Untersuchungen.}

Unsere Untersuchungen wurden ebenfalls nach der Bangschen Methode ausgeführt. Der Harnstoff wurde nicht mitbestimmt. Die Untersuchungen erstrecken sich auf Schwangere, Wöchnerinnen ohne und mit Erkrankungen und Eklampsien.

1. Normale Gravidae und Wöchnerinnen: Die Fälle von Gravidae und Wöchnerinnen, die uns zur Untersuchung zur Verfügung standen, zeigen in keiner Weise eine Erhöhung des Rest-N ante partum und sub partu. Auch post partum steigt der Rest-N nie höher als bis zur oberen Grenze des Normalen.

Wir finden also ein völlig normales Verhalten sowohl der Leber im Eiweißumsatz als auch der Niere, was die Fähigkeit anbelangt, harnfähige Stoffe auszuscheiden.

2. Gravidae und Wöchnerinnen mit verschiedenen Erkrankungen: Sie zeigen je nach der Erkrankung verschiedene Werte. Zwei Nephropathien zeigen in der Schwangerschaft Werte nach der oberen Grenze des Normalen hin.

Ein Fall von Nephropathie, am Tage des Partus auf den Rest- $\mathrm{N}$ Gehalt untersucht, zeigt eine leichte Erhöhung des Rest-N 0,055\%. Zudem handelt es sich hier um eine langdauernde Geburt, was evtl. auch einen Einfluß auf die Höhe des Rest-N-Spiegels hat. 
Wochenbettswerte von zwei anderen Nephropathien sind erhöht. So besonders 1 Fall (Schläfer) bis $0,051 \% 1$ Monat post partum. Es unterliegt wohl keinem Zweifel, daß in diesen Fällen die Erhöhung des Rest-N nephrogener Ursache ist.

Ein Fall von Chorea zeigt noch erhöhten. Wert in der Schwangerschaft. - Die übrigen Schwangerschaftswerte sind normal.

3. Eklampsicn: Eklampsien zeigen kurz ante, am Tage der Geburt und kurz nach der Geburt eine deutliche Steigung des Rest-N-Wertes.

Der Wert ante partum kann schon ganz erhebliche Höhe erreichen, so 0,083 einen Tag ante partum (Lipp).

Sub partu, d. h. am Tage des Partus sind die Werte stets erhöht, sie schwanken zwischen Werten an der oberen Grenze des Normalen und über das Normale hinaus erhöhten pathologisehen Werten.

Unser Mittelwert für die Spiegelhöhe sub partu beträgt 0,055\%, wobei der höchste Wert $0,076 \%$, der tiefste $0,039 \%$ beträgt.

Vergleicht man die Zahl der Anfälle mit der Höhe des Rest-NSpiegels am Tage der Geburt, so kann man sagen, daß alle Fälle, die nicht einen erhöhten Spiegel haben, nur einen eklamptischen Anfall erlitten haben.

Es liegt indessen auch ein Fall vor (Swoboda), wo bei einem Anfall der Spiegel auf $0,063 \%$ stieg. Es läBt sich indessen daraus nichts Sicheres auf einen Zusammenhang über Spiegelhöhe und Anzahl der Anfälle schließen. - Im Wochenbett besteht kurz post partum noch eine Erhöhung des Rest-N-Spiegels. Er sinkt indessen bald früher, bald später und erreicht schließlich wieder die Norm. Die vielen Bestimmungen im Wochenbett zeigen diese Rückkehr zur Norm in schön. ster Weise an (Schäfer, Horchler). Vgl. S. 93-95, Tab. IV c.

Tabelle IVa. Reststickstoffbestimmungen.

Gravidae und Wöchnerinnen.

\begin{tabular}{llll}
$\quad$ Name & \multicolumn{1}{c}{ Datum } & Rest-N & Bemerkungen \\
Schwank & 9. IX. 1918 & 0,036 & mens. 10 \\
Werner & 5. IX. 1918 & partus & \\
& 9. IX. 1918 & 0,040 & \\
Pulch & 6. IX. 1918 & partus & \\
& 9. IX. 1918 & 0,029 & \\
Beck & 11. IX. 1918 & 0,032 & mens. 10 \\
& 16. IX. 1918 & 0,035 & Blutentn. v. Ausst. d. \\
Schwarzmann & 10. IX. 1918 & partus & Placenta; sub partu \\
& I6. IX. 1918 & 0,037 & \\
Wiegand & 18. IX. 1918 & 0,029 & sub partu \\
Kable & 20. IX. 1918 & 0,039 & Grav. \\
Sander & 20. IX. 1918 & 0,032 & sub partu \\
Jacob & 23. IX. 1918 & 0,030 & am Tage d. Partus
\end{tabular}




\section{Tabelle IV b.}

Gravidae und Wöchnerinnen mit versehiedenen Erkrankungen. Name Datum

Rest-N

Bemerkungen

Weiss

25. VI. 1919

0,037 a. p.

neph, in grav.

Krämer

21. II. 1920

0,038 a. p.

neph. grav. mens. 5

Palitza

15. VII. 1919

0,031 a. $\mathrm{p}$.

Hydrops mens 9

Bienmüller

8. I. 1920 0,029 a. p. 4 . W.

Heuss

20. II. 1920

0,044 a. p.

icterus in grav.

Lehmkuhl

4. III. 1920

0,034 a. p.

Chorea mens. 8; Bl.-Dr. 141

4. III. 1920

Chorea; Bl.-Dr. 110 sectio

Trumpp

3. VI. 1919

F. partus

11. VI. 1919

0,037

Vit. Cordis

Hörlein

14. IV. 1920

F. partus

3. V. 1920

0,042

17. V. 1920

0,037

Dörr

27. XI. 1919

partus

27. XI. 1919

0,055

29. XI. 1919

Schläfer

3. V. 1920

10. V. 1920

F. partus $30 . W$.

0,045

0,051

1. VI. 1920

14. VI. 1920

0,044

24. VI. 1920

0,042

6. VII. 1920

0,036

Chron. neph. Bl. 110

neph. in grav.; Bl.-Dr. 150;

Eiw. $12 \%$

Bl.-Dr. 140

Tabelle IVe. Eklampsien.

Name Datum Eiweißg. d. Urins

Rest-N

Blutdruck

Weiler

29. III. 1920

$+\frac{1}{i}$

F. partus 39. W.

160

1. IV. 1920

0,057

8. IV. 1920

0,046

20. V. 1920

0,039

Heuser

10. V. 1920

11. V. 1920

20. V. 1920

31. V. 1920

6. VI. 1920

9. VI. 1920

$12 \%$

145

23. IV. 1920

Englert

$\begin{array}{ll}2 \% & 0,038 \\ & 0,050 \\ 1 \% & \text { F. partus 36. W. } \\ & 0,044\end{array}$

ph. in grav.; Bl.-Dr. 110;

Eiw. $1 / 2 \%$

Heeb

22. IV. 1920

$4 \%$

0,048

150

sectio; exitus sub op. 32.W.

22. IV. 1920

partus

5. V. 1920

Swobada

11. IV. 1920

11. IV. 1920

20. IV. 1920

24. IV. 1920

$\begin{array}{ll}\text { pos. } & 0,040 \\ & 0,034\end{array}$

3. V. 1920

$14^{\circ} \%$ partus

130

$1 / 2 \% 00$

0,063

0,038

0,037 


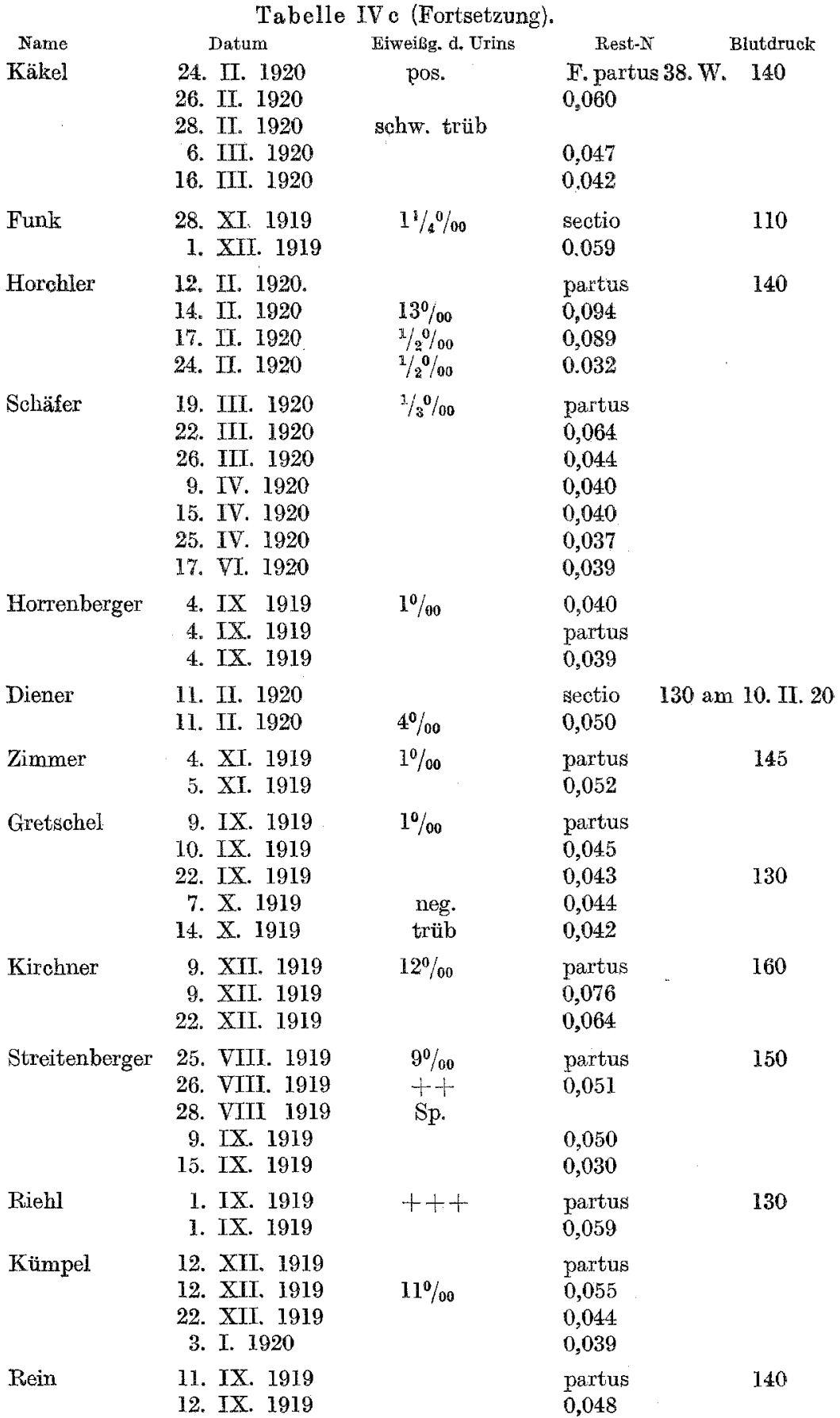




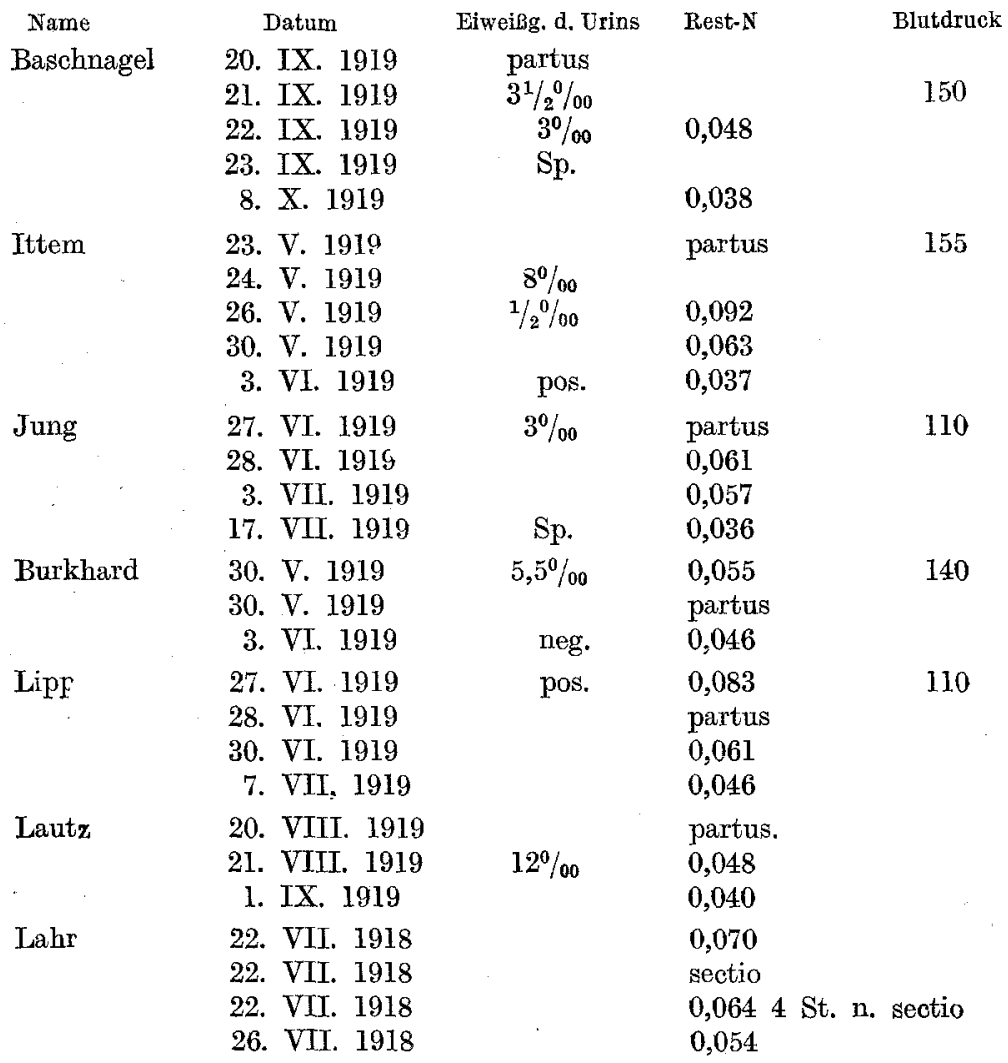

\section{Zusammenfassung.}

Überblickt man noch einmal, wie in der Gravidität, sub partu, im Wochenbett und bei Eklamptischen die Leber bei den verschiedenen Funktionsprüfungen reagiert, so kommt man kurz zu folgenden Schlüssen:

In der Gravidität besteht ein normaler, eher tiefer Blutzuckerspiegel. Infusionen von Traubenzucker werden restlos in der normalen Zeit gespeichert. - Injektionen von Adrenalin zeigen auf den Blutzuckerspiegel keinen anderen Einfluß wie bei Nichtgraviden. - Der Urobilingehalt ist in der Schwangerschaft vermehrt, und zwar nur in der zweiten Hälfte bei vielen Fällen.

Eine Injektion von Adrenalin zeigt keinen Einfluß auf den Urobilingehalt des Urins. Der Rest-N ist nicht erhöht. Die Vermehrung des Urobilins für viele Fälle ist das einzige Kriterium einer Leberinsuffizienz in der Schwangerschaft.

In der Zeit unmittelbar vor der Geburt, während und unmittelbar nach der Geburt bestehen besondere Verhält. nisse. Der Blutzuckerspiegel erhebt sich wieder zur Norm und geht 
sub partu in eine Hyperglykämie über, die kurz post partum noch andauert.

Das Speicherungsvermögen der Leber kurz ante, sub und post partum ist noch nicht mit Sicherheit festgestellt. Zwei Untersuchungen einen Tag post partum zeigen leichte Verzögerungen im Speicherungsvermögen. Es ist gut möglich, daß sub partu das Speicherungsvermögen für intravenös infundierten Traubenzucker herabgesetzt ist.

Injektionen von 0,75 Adrenalin haben kurz ante, am Tage des Partus und kurz post partum einen ganz besonderen Einfluß auf das Mobilisierungsvermögen von Traubenzucker in der Leber. Kurz vor und nach dem Partus finden wir eine verminderte Anspruchsfähigkeit der Leber auf Adrenalin (wobei offen bleibt, ob der Sympathicus oder die Leberzelle eine herabgesetzte Anspruchsfähigkeit besitzen). Am Tage des Partus versagt die Leber völlig auf eine Adrenalininjektion hin. Der Spiegel sinkt sogar bis zu Werten unterhalb des Wertes vor Injektion.

Weder Urobilingehalt an und für sich, noch der Einflub einer Adrenalininjektion auf den Urobilingehalt des Urins weisen Besonderheiten auf. Das Urobilin ist wie in der übrigen Schwangerschaft vermehrt.

Der Rest-N zeigt nicht erhöhte Werte.

$Z$ usa m mengefa $B$ t zeigt die Leber regelmäßig sub partu folgende Funktionsstörungen:

1. Hy perglykämie, d. h. erhöhten Blutzucker - Nüchternspiegel.

2. Wahrscheinlich verzögertes Speicherungsvermögen, d.h. Unvermögen, eine intravenös infundierte Zuckerlösung innerhalb spätestens einer Stunde zu speichern.

3. Vermindertes oder a ufgehobenes Mobilisierungsver mögen des Traubenzuckers. Verminderte oder a ufgehobene Fähigkeit der Leber a uf einen Adrenalinreiz hin Zucker ins Blut hin aususchütten.

4. Vermehrtes Urobilin.

Es ist möglich, daß Speicherungsvermögen und Mobilisierungsvermögen für Traubenzucker Hand in Hand gehen. Liegt das Speicherungsvermögen danieder, dann ist auch das Mobilisierungsvermögen geschädigt.

Wir sehen also kurz vor der Geburt eine große Umwälzung im Sinne zunehmender Insuffizienz der Leber vor sich gehen. Bestände nicht die Möglichkeit, daß das Auftreten dieser Insuffizienzerscheinungen den Anstoß zum Partus bedingt?

Im Wochenbett sehen wir die Herstellung normaler Verhältnisse vor sich gehen. Die Leber wird wieder suffizient, bald rascher, bald langsamer. - Der Blutzuckerspiegel sinkt, das Speicherungs- und Mobilisierungsvermögen kehrt zur Norm zurück, das Urobilin verschwindet aus dem Urin. 
Verschiedene Erkrankungen haben verschiedenen Einfluß auf die Vorgänge der Leber. Es gehören diese Vorgänge in das Gebiet der inneren Medizin. Erwähnen möchte ich nur noch die bei Leberkrankheiten in ganz gleicher Weise wie zur Zeit des Partus auftretenden Leberfunktionsstörungen. Ferner möchte ich die weniger untersuchten Graviditätstoxikosen erwähnen, wo wir ebenfalls Leberfunktionsstörungen feststellen können.

Bei Eklamptischen ist der Nüchternblutzuckerspiegel stark erhöht. Das Speicherungsvermögen für Traubenzucker hat außerordentlich gelitten. Hingegen läßt sich über das Mobilisierungsvermögen noch nichts Eindeutiges aussagen. Es wurden nur Untersuchungen im Wochenbett angestellt. Auch der Urobilingehalt läßt keine besonderen Schlüsse zu. Hingegen ist der Rest-N in ganz typischer Weise erhöht. Untersuchungen über die Größe der Harnstoffquote müssen entscheiden, ob diese Rest-N-Vermehrung hepatogener oder nephrogener Ursache ist.

Aus allen diesen Ausführungen geht hervor, daß der Begriff ,Schwangerschaftsleber" im funktionellen Sinne absolut zu Recht besteht. Nur müssen wir ihn zeitlich begrenzt fassen. - Eine Schwangerschaftsleber in ausgesprochenem Sinn besteht nur kurz ante, sub und post partum.

Graviditätstoxikosen, worunter die Eklampsie die hervorragendste Rolle spielt, zeigen ebenfalls in verschiedenem Maße gestörte Leberfunktion.

\section{Literatur.}

Hofbauer, Zeitschr. f. Geburtsh. u. Gynäkol. 61. 1907. - Hofbauer, Arch. f. Gynäkol. 93. 1911. - Schickele, Arch. f. Gynäkol. 92. 1910 - Heinrichsdorff, Zeitsch. f. Geburtsh. u. Gynäkol. \%. 1912. - Bang, Mikromethoden. Bergmann, 1920. - Bang, Der Blutzucker. Bergmann, 1913. -Benthin, Zitschr. f. Gaburtsh. u. Gynäkol. 69. 1911. - Neubauer und Novac, Dtsch. med. Wochenschr. 1911, Nr. 49. - R yser, Dtsch. Arch. f. klin. Med. 118. - Grote, Zentralbl. f. inn. Med. 1918, Nr. 23. - Brosamlen und Sterkel, Dtsch. Arch. f. klin. Med. 130. 1919. - Stra ub, Dtsch. Arch. f. klin. Med. 11\%. - Loew y, Berl. klin. Wochenschr. 1917, Nr. 41. - Jakobsen, Biochem. Zeitschr. 56. 1913. - Kahler, Zeitschr. f. angew. Anat. u. Konstitutionslehre. - Bang, Biochem. Zeitschr. 72. 1916. - Seitz, Handbuch Döderlein. - Blum, Dtsch. Arch. f. klin. Med. 91. 1901. - Landau, Zeitschr. f. klin. Med. \%9. 1916. Fischler, Phys. und athologie der Leber. Jul. Springer, 1916. - Schlesinger, Dtsch. med. Wochenschr. 1903. - Müller, Über Ikterus. Verhandl. d. Schles. Ges. f. vaterl. Kultur 1892. - Hop pe - Seyler, Handbuch der phys. und pathologisch-chemischen Analyse. - Gri m m, Über Urobilin im Harn. Virchows Arch. f. pathol. Anat. u. Physiol. 194. - Merletti, Zentralbl. f. Gynäkol. 1902, Nr. 16. - Guggisberg, Monatsschr. f. Geburtsh. u. Gynäkol. 45, 334. 1917. - Pfan nhaser und Pfitzer, Münch. med. Wochenschr. 1913, S. 2155. 